\title{
Colloidal ReO3 Nanocrystals: Extra Re d-Electron Instigating a Plasmonic Response
}

\author{
Sandeep Ghosh, Hsin-Che Lu, Shin Hum Cho, Thejaswi Maruvada, Murphie C. Price, Delia Milliron
}

Submitted date: 02/07/2019 - Posted date: 03/07/2019

Licence: CC BY-NC-ND 4.0

Citation information: Ghosh, Sandeep; Lu, Hsin-Che; Cho, Shin Hum; Maruvada, Thejaswi; Price, Murphie C.; Milliron, Delia (2019): Colloidal ReO3 Nanocrystals: Extra Re d-Electron Instigating a Plasmonic Response. ChemRxiv. Preprint.

Rhenium (+6) oxide ( $\mathrm{ReO} 3)$ is metallic in nature, which means it can sustain localized surface plasmon resonance (LSPR) in its nanocrytalline form. Herein, we describe the colloidal synthesis of nanocrystals (NCs) of this compound, through a hot-injection route entail- ing the reduction of rhenium $(+7)$ oxide with a long chain ether. This synthetic protocol is fundamentally different from the more widely em- ployed nucleophilic lysing of metal alkylcarboxylates for other metal oxide NCs. Owing to this difference, the NC surfaces are populated by ether molecules through an L-type coordination along with covalently bound (X-type) hydroxyl moieties, which enables easy switching from nonpolar to polar solvents without resorting to cumbersome ligand exchange procedures. These as-synthesized NCs exhibit absorption bands at around $590 \mathrm{~nm}(\approx 2.1 \mathrm{eV})$ and $410 \mathrm{~nm}(\approx 3$ $\mathrm{eV}$ ), which were respectively ascribed to their LSPR and interband absorptions by Mie theory simulations and Drude modeling. The LSPR response arises from the oscillation of free electron density created by the extra Re d-electron per ReO3 unit in the NC lattice, which resides in the conduction band. Further, the LSPR contribution facilitates the observation of dynamic optical modulation of the NC films as they undergo progressive electrochemical charging via ion (de)insertion. Ion (de)insertion leads to distinct dynamic optical signatures, and these changes are reversible in a wide potential range depending on the choice of the ion (lithium or tetrabu-tylammonium). Nanostructuring in $\mathrm{ReO} 3$ and the description of the associated plasmonic properties of these NCs made this optical modulation feasible, which were hitherto not reported for the bulk material. We envisage that the synthetic protocol described here will facilitate further exploration of such applications and fundamental studies of these plasmonic NCs

File list (2)

Manuscript.pdf (3.41 MiB)

view on ChemRxiv - download file

Supporting information.pdf (2.00 MiB)

view on ChemRxiv - download file 


\title{
Colloidal $\mathrm{ReO}_{3}$ Nanocrystals: Extra Re d-electron instigating a plasmonic re- sponse
}

\author{
Sandeep Ghosh ${ }^{\dagger}, \mathrm{Hsin}-\mathrm{Che} \mathrm{Lu}^{\dagger}$, Shin Hum Cho ${ }^{\dagger}$, Thejaswi Maruvada ${ }^{\dagger}$, Murphie C. Price ${ }^{\dagger}$, and Delia J. Milliron ${ }^{+* *}$
}

${ }^{+}$McKetta Department of Chemical Engineering, The University of Texas at Austin, Austin, Texas 78712-1589, United States

\begin{abstract}
Rhenium (+6) oxide $\left(\mathrm{ReO}_{3}\right)$ is metallic in nature, which means it can sustain localized surface plasmon resonance (LSPR) in its nanocrytalline form. Herein, we describe the colloidal synthesis of nanocrystals (NCs) of this compound, through a hot-injection route entailing the reduction of rhenium $(+7)$ oxide with a long chain ether. This synthetic protocol is fundamentally different from the more widely employed nucleophilic lysing of metal alkylcarboxylates for other metal oxide NCs. Owing to this difference, the NC surfaces are populated by ether molecules through an L-type coordination along with covalently bound (X-type) hydroxyl moieties, which enables easy switching from nonpolar to polar solvents without resorting to cumbersome ligand exchange procedures. These as-synthesized NCs exhibit absorption bands at around $590 \mathrm{~nm}(\approx 2.1 \mathrm{eV})$ and $410 \mathrm{~nm}(\approx 3 \mathrm{eV})$, which were respectively ascribed to their LSPR and interband absorptions by Mie theory simulations and Drude modeling. The LSPR response arises from the oscillation of free electron density created by the extra Re d-electron per $\mathrm{ReO}_{3}$ unit in the NC lattice, which resides in the conduction band. Further, the LSPR contribution facilitates the observation of dynamic optical modulation of the NC films as they undergo progressive electrochemical charging via ion (de)insertion. Ion (de)insertion leads to distinct dynamic optical signatures, and these changes are reversible in a wide potential range depending on the choice of the ion (lithium or tetrabutylammonium). Nanostructuring in $\mathrm{ReO}_{3}$ and the description of the associated plasmonic properties of these NCs made this optical modulation feasible, which were hitherto not reported for the bulk material. We envisage that the synthetic protocol described here will facilitate further exploration of such applications and fundamental studies of these plasmonic NCs.
\end{abstract}

\section{INTRODUCTION}

Rhenium(VI) oxide $\left(\mathrm{ReO}_{3}\right)$ exhibits characteristics of a good metallic conductor, ${ }^{1-2}$ unlike the wide band-gap semiconducting nature that is generally exhibited by other stoichiometric metal oxides. The bulk metallic nature of this lustrous red oxide was confirmed by absolute reflectance measurements by Feinleib et al. ${ }^{1}$ The dielectric constant was found to exhibit free electron characteristics below the sharp plasma edge of $2.1 \mathrm{eV}$ and interband transitions dominate the optical spectrum at higher energies. Metallic conductivity has been demonstrated through resistivity and Hall effect measurements where the negative sign of the Hall constant (measured as -3.28 $\times 10^{-4} \mathrm{~cm}^{3} \mathrm{C}^{-1}$ at $300 \mathrm{~K}$ ) signified electrons as the predominant charge carriers, ${ }^{3}$ with an electron mean free path of $89 \AA$ at $300 \mathrm{~K}^{4} \mathrm{ReO}_{3}$ exhibits conductivity of a typical metallic conductor, an order of magnitude smaller than that of copper, but roughly the same as titanium and chromium.

Incomplete d-shells of transition metals bestow interesting properties in compounds they constitute and, in case of $\mathrm{ReO}_{3}$, where $\mathrm{Re}^{6+}$ is a $\mathrm{d}^{1}$ system (outer electron configuration of rhenium being $\left.5 d^{5} 6 s^{2}\right)$ this confers a metallic character, as has been demonstrated by Ferretti et al. ${ }^{2}$ The metallic conductivity has been ascribed to the strong hybridization between $\mathrm{Re} 5 \mathrm{~d}$ and $\mathrm{O} 2 \mathrm{p}$ atomic orbitals, which leads to significant broadening of the $5 \mathrm{~d}$ conduction band. ${ }^{5-7}$ That Re $5 \mathrm{~d}$ states constitute the conduction band has been confirmed by $\mathrm{X}$-ray photoemission analysis, ${ }^{7}$ and the Fermi level location in the $5 \mathrm{~d}$ manifold has been deduced by nuclear magnetic resonance (NMR) spectroscopy. ${ }^{7-8}$

Despite the metallic electronic behavior, the properties of the $\mathrm{ReO}_{3}$ lattice are not typical of that of metals, as suggested by the crystal structure. ${ }^{9} \mathrm{ReO}_{3}$ crystallizes in the simple cubic structure $\mathrm{Pm} \overline{3} \mathrm{~m}$, which is not a close-packed metallic phase. ${ }^{5}$ In fact, the structure of $\mathrm{ReO}_{3}$ is best described as the perovskite $\mathrm{ABO}_{3}$ structure with the larger cationic A-sites remaining vacant.
Being a metallic solid with a considerable free electron density, $\mathrm{ReO}_{3}$ should exhibit plasmonic characteristics (collective oscillations of these free electrons) and, at the nanoscale, localized surface plasmon resonance (LSPR). However, the lack of a robust synthesis protocol yielding discrete NCs with colloidal stability made a proper description of the plasmonic response an elusive endeavor. Previous synthetic efforts on $\mathrm{ReO}_{3} \mathrm{NCs}$ include solvothermal and sol gel strategies, ${ }^{10-12}$ and incorporating them in polymer matrices, ${ }^{13}$ where the LSPR response of nanostructured $\mathrm{ReO}_{3}$ was treated rather phenomenologically. Recent colloidal synthetic efforts include that by Jeong et al., who used thermolysis of a mixture of $\operatorname{Re}(+7)$ oxide and oleic acid to form NCs where Re atoms were in mixed oxidation states. ${ }^{14}$ This particular report goes on to show the inherent difficulty in devising a colloidal route for plasmonic $\mathrm{ReO}_{3} \mathrm{NCs}$ at a fixed +6 oxidation state. The availability of a large number of oxidation states $(+3$ to +7$)$ for Re and the lack of suitable precursors with Re in the appropriate oxidation state and coordination number are the chief reasons for the synthetic difficulty. This makes it particularly challenging to adapt the usual synthetic route for metal oxide NCs for this case - that of nucleophilic lysing of metal alkylcarboxylate complexes, which also explains the lack of relevant literature so far.

Here, we describe a colloidal hot injection synthesis approach for producing $\mathrm{ReO}_{3} \mathrm{NCs}$ where a nonaqueous "soft" reduction of rhenium (+7) oxide was employed. Ether molecules containing long carbon chains or multiple ether functionalities were used as reducing agents in addition to their roles as solvents in the injection solution and as the eventual surface ligands. The as-prepared NCs exhibit a mixed ligand shell with L-type coordination by the long carbon chain ether molecules and covalently bound hydroxyl groups (Xtype), as confirmed by NMR and Fourier transform infrared (FTIR) spectroscopy. The surface hydroxyl groups were produced during the reduction of the Re-precursor. While the ether molecules imparted colloidal stability to the NCs, they are weak Lewis bases with 
labile surface coordination and hence can be easily stripped off of the NC surfaces by washing with polar solvents. In fact, this mixed ligand shell leads to easy transfer of these NCs from a nonpolar solvent to a polar medium simply through multiple purification cycles. Different from other colloidal NCs, ${ }^{15}$ an arduous ligand exchange procedure involving post-synthetic treatment with harsh chemical moieties can be avoided in this case. This ease of solution processibility facilitates the investigations of these NCs both in isolation (as in dispersion) as well as in interacting ensembles (as in film fabrication from polar solvents).

The optical extinction of the as-synthesized NCs exhibits an absorption band around $590 \mathrm{~nm}(\approx 2.1 \mathrm{eV})$ ascribable to an LSPR response analogous to other metallic NCs, in addition to an interband absorption around $410 \mathrm{~nm}(\approx 3 \mathrm{eV})$. The calculated absorption spectrum by Mie solutions to Maxwell equations from bulk dielectric constants of $\mathrm{ReO}_{3}$ agree with our observations and a Drude treatment of the LSPR band yields appropriate free electron densities in the NCs. The free electron (LSPR) and the bound electron (interband) contributions to the extinction spectrum have thus been demarcated. Furthermore, the LSPR peak energy was found to be sensitive to the dielectric constant of the surrounding medium, which can serve as a useful tool in molecular sensing. The LSPR response broadens when the NCs are deposited as films and was modulated through EC ion (de)insertion. These optical modulations with lithium ( $\mathrm{Li}$ ) and tetrabutylammonium (TBA) ions were reversible over a wide potential range and are facilitated by the nanostructuring and thus from a clear understanding of the LSPR mode in $\mathrm{ReO}_{3} \mathrm{NCs}$.

\section{EXPERIMENTAL SECTION}

Materials. Rhenium $(+7)$ oxide $\left(\operatorname{Re}_{2} \mathrm{O}_{7}, \geq 99.9 \%\right)$, tetraglyme (TGY, $\geq 99 \%$ ) dioctyl ether (DOE, 99\%), lithium bis-trifluoromethanesulfonimidate (Li-TFSI), tetrabutylammonium perchlorate $\left(\mathrm{TBA}-\mathrm{ClO}_{4}, \geq 99.0 \%\right)$, 1-octadecene (ODE, technical grade, 90\%), and anhydrous propylene carbonate (PC, 99.7\%), dimethyl carbonate (DMC, $\geq 99.0 \%$ ), chloroform, hexane and isopropanol were purchased from Sigma-Aldrich. All chemicals were used as received.

Synthesis of $\mathrm{ReO}_{3} \mathrm{NCs}$. All syntheses were undertaken using standard Schlenk line techniques with nitrogen filled glovebox as an aid. In a typical synthesis, a mixture of DOE $(5 \mathrm{ml})$ and ODE $(5 \mathrm{ml})$ were loaded in a three-neck round bottom flask and degassed under vacuum at $100^{\circ} \mathrm{C}$ for $1 \mathrm{~h}$. The flask was then backfilled with nitrogen and the temperature was raised to the desired reaction temperature $\left(100-260{ }^{\circ} \mathrm{C}\right)$. A separate solution of $\mathrm{Re}_{2} \mathrm{O}_{7}$ powder $(25 \mathrm{mg}, 0.05$ $\mathrm{mmol})$ in TGY $(400 \mu \mathrm{l})$, prepared in a glovebox, was then injected into the reaction flask. The colorless transparent solution immediately changed into a blue-black solution, signifying the formation of $\mathrm{ReO}_{3} \mathrm{NCs}$. The heating mantle was then removed and the flask was cooled with an air jet. The final dark blue solution was then washed with a chloroform/isopropanol mixture. The washing procedure involved precipitating the NCs out of the chloroform dispersion using isopropanol as the anti-solvent, and discarding the supernatant after centrifuging the suspension at $4500 \mathrm{rpm}$ for $5 \mathrm{~min}$. The NC pellet was redispersed in chloroform and this washing procedure was repeated twice. The NCs were finally redispersed in chloroform.

Notes: (a) $\mathrm{Re}_{2} \mathrm{O}_{7}$ is hygroscopic and reacts with water to form perrhenic acid $\left(\mathrm{HReO}_{4}\right)$ - it should be stored in an inert atmosphere (like a nitrogen filled glovebox). (b) $\operatorname{Re}_{2} \mathrm{O}_{7}$ is soluble in TGY, but the initially colorless solution turns brown over time, and hence should be freshly prepared before use.

$\mathrm{X}$-ray Diffraction (XRD) analysis. Concentrated dispersions of the NCs were drop cast onto silicon substrates for XRD measurements. Data collection was performed on Rigaku MiniFlex 600 Xray diffractometer using $\mathrm{Cu} \mathrm{Ka}$ radiation $(1.5418 \AA$ ) . The DebyeScherrer method was used to calculate the sizes of the deposited NCs, using the following equation:

$$
\tau=\frac{\mathrm{K} \lambda}{\beta \cos \theta}
$$

Here, $\lambda$ is the $\mathrm{X}$-ray wavelength $(0.15418 \mathrm{~nm}), \beta$ is the line broadening, and $\theta$ is the Bragg angle of the XRD peak. The line broadening and the Bragg angle were obtained for the (210) peak by fitting it with a pseudo-Voigt lineshape. The line broadening was corrected for instrumental broadening by $\beta=\left(w_{\text {exp }}^{2}-w_{\text {ins }}^{2}\right)^{1 / 2}$, where $w_{\text {exp }}$ is the measured full width at half maximum (FWHM) and $w_{\text {ins }}$ is the FWHM measured from $\mathrm{LaB}_{6}$ powder standard $\left(0.136^{\circ}\right)$. The Scherrer constant K was set to $0.9 \pm 0.045$ as per Langford and Wilson. ${ }^{16}$

Steady State UV-Vis-NIR Extinction Spectroscopy. Diluted solutions of NCs in chloroform were loaded in quartz cuvettes of 1 $\mathrm{cm}$ path-length and their optical extinction spectra were recorded in a Varian Cary 5000 UV-Vis-NIR absorption spectrophotometer in the wavelength range of $300-2200 \mathrm{~nm}$.

Transmission electron microscopy (TEM) analysis. Sample preparation involved drop-casting dilute NC dispersions onto carbon-coated 400 mesh copper grids (Ted Pella). A JEOL 2010F microscope equipped with a CCD camera and a Schottky field emission gun operating at $200 \mathrm{kV}$ was used to acquire the high-resolution transmission electron microscopy (HRTEM) images and selectedarea electron diffraction (SAED) patterns. Analysis and signal processing of the SAED patterns (including beam-stop removal, centering and eventual azimuthal integration coupled with background subtraction) was performed using the PASAD software. ${ }^{17}$

FTIR spectroscopy. NC dispersions were dropcast onto undoped silicon substrates and the spectra were recorded in transmission in a Bruker VERTEX 70 spectrometer, at $4 \mathrm{~cm}^{-1}$ scan resolution.

NMR spectroscopy. Deuterated solvents, chloroform-d $\left(\mathrm{CDCl}_{3}, 99.96\right.$ atom \% D) and $N, N$-dimethylformamide-d ${ }_{7}(\mathrm{DMF}-$ $\mathrm{d}_{7}, \geq 99.5$ atom $\% \mathrm{D}$ ), were purchased from Sigma-Aldrich. NC samples were subjected to multiple washing cycles $(\geq 10)$ to ensure clean samples for spectroscopy. The NCs were then dried under a strong nitrogen flow to remove the residual non-deuterated solvents, followed by redispersing them in the desired deuterated solvent. For each measurement, $40 \mathrm{mg}$ of the NCs were dispersed in 1 $\mathrm{ml}$ of the deuterated solvent. Care was taken to avoid the presence of moisture in the NMR samples. Special screw-capped NMR tubes (Norell) were rinsed with chloroform and dried under vacuum in the glovebox antechamber prior to loading the sample. Fresh ampoules of deuterated solvents were used for every measurement and the final stages of the sampling were performed in the glove box. The spectra were acquired using Agilent/Varian MR-400 (operating at ${ }^{1} \mathrm{H}$ frequency of $400 \mathrm{MHz}$ ) or VNMRS 600 (operating at ${ }^{1} \mathrm{H}$ frequency of $600 \mathrm{MHz}$ ) spectrometers, with sample temperature set at $300 \mathrm{~K}$. Solvent signals were used as reference for analysis. Spectra were recorded in $1 \mathrm{D}{ }^{1} \mathrm{H}$ and $2 \mathrm{D}{ }^{1} \mathrm{H}-{ }^{13} \mathrm{C}$ correlation heteronuclear single quantum coherence (HSQC) modes.

Elemental analysis. The concentrations of NC dispersions were determined through inductively coupled plasma-optical emission spectroscopy (ICP-OES) measurements on a Varian 720-ES ICP Optical Emission Spectrometer. The samples were prepared by digesting them in aqua regia for $24 \mathrm{~h}$ and then diluting by a known factor. 
Spectroelectrochemical (SEC) measurements. The multiply washed hydroxyl-terminated NCs were drop cast onto ITO-coated glass substrates for SEC measurements. The EC and in situ optical measurements were performed with an EC workstation (Bio-logic VMP3 potentiostat) and an ASD LabSpec 4 Std-Res VIS/NIR spectrometer in an argon filled glovebox. Our home-built setup employs a three-electrode configuration for EC measurements and consists of a wide quartz cuvette housing the electrolyte with the NC film (as the working electrode), the counter, and the reference electrodes all immersed into it. The spectra were recorded in the transmission mode at various potentials, with the source and detector fiber optic cables of the spectrometer positioned in a perpendicular orientation to the NC film. The EC experiments performed include chronoamperometry $(\mathrm{CA})$ and cyclic voltammetry $(\mathrm{CV})$. For the Li-ion measurements, a $\mathrm{Li}$ foil served both as the reference and the counter electrodes and $1 \mathrm{M} \mathrm{Li-TFSI}$ in TGY as the electrolyte for the potential range of 1-4 V. On the other hand, for TBA-ion based measurements, a Pt foil was used as a counter electrode, a commercial fritted $\mathrm{Ag} / \mathrm{Ag}^{+}$cell as the reference electrode and $0.5 \mathrm{M} \mathrm{TBA}-\mathrm{ClO}_{4}$ in PC as the electrolyte. The reference electrode was immersed in a $0.01 \mathrm{M}$ $\mathrm{AgNO}_{3}$ and $0.5 \mathrm{M}$ TBA-ClO ${ }_{4}$ in $\mathrm{PC}$ solution and was calibrated to a $\mathrm{Li}$ foil. The open-circuit potential (OCP) of the NC films were taken as the potential recorded when the film was dipped into the electrolytes. The OCPs recorded were $2.6 \mathrm{~V}$ (vs $\mathrm{Li} / \mathrm{Li}^{+}$) and $-1.2 \mathrm{~V}$ (vs $\left.\mathrm{Ag} / \mathrm{Ag}^{+}\right)$. The spectra were recorded after current reached a steady state at a given potential, typically less than $2 \mathrm{~min}$. To ensure a steady current, the films were held at a given potential for $5 \mathrm{~min}$ before every measurement. CV was performed at $10 \mathrm{mV} / \mathrm{s}$ scan rate.

X-ray photoelectron spectroscopy (XPS). NCs were drop-cast onto silicon substrates and a Kratos Axis Ultra DLD spectrometer with a monochromatic $\mathrm{Al} \mathrm{Ka}$ source $(1486.6 \mathrm{eV})$ was used for the measurements. An analyzer pass energy of $80 \mathrm{eV}$ was employed for wide scans while a pass energy of $20 \mathrm{eV}$ was used for the high-resolution narrow region scans, with steps of $0.1 \mathrm{eV}$. The pressure in the analysis chamber was maintained at around $10^{-9}$ torr and the spectra were acquired at a photoelectron take-off angle of $0^{\circ}$ with respect to the surface normal. Data was analyzed with CasaXPS software using the Kratos relative sensitivity factor library. The binding energy (BE) scale was internally referenced to the $\mathrm{C} 1 \mathrm{~s}$ peak $(\mathrm{BE}$ for $\mathrm{C}-\mathrm{C}=$ $284.8 \mathrm{eV})$.

Special care was exercised for the samples at different stages of EC (dis)charging. Plastic tweezers were used to handle the NC films and the excess electrolyte was washed off using DMC. These films were then dried and loaded on a metallic XPS stage, where the glass portion of the ITO substrate acted as the insulating layer between the NC film and the stage. This ensured that the films remained at the same level of original charge. The XPS stage with the samples were then loaded into an air-free transfer capsule inside the argon filled glove box. This capsule was then attached to the spectrometer and the stage was transferred inside the XPS analysis chamber without exposing the samples to the ambient atmosphere.

\section{RESULTS AND DISCUSSION}

Synthesis and characterization of the NCs. Scheme 1 below shows the reaction that was employed in synthesizing the $\mathrm{ReO}_{3}$ NCs. In this synthesis, phase pure NCs were formed through a colloidal approach wherein the rhenium precursor, rhenium $(+7)$ oxide $\left(\mathrm{Re}_{2} \mathrm{O}_{7}\right)$, was dissolved in dry TGY in an inert atmosphere. $\mathrm{Re}_{2} \mathrm{O}_{7}$ being hygroscopic, it reacts easily with adventitious moisture to form perrhenic acid, ${ }^{18-19}$ which is detrimental to the NC synthesis. Hence, it is important that this precursor be stored and the solution be pre- pared in an inert atmosphere like a nitrogen-filled glove box. Thereafter, this $\mathrm{Re}_{2} \mathrm{O}_{7}$-TGY solution was injected into hot DOE, in admixture with $\mathrm{ODE}$, to produce the $\mathrm{ReO}_{3} \mathrm{NCs}$. The temperature at which the injection was performed varied between 100 and $260^{\circ} \mathrm{C}$.

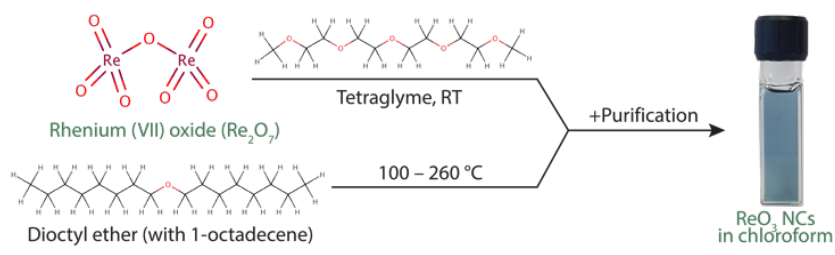

Scheme 1. Colloidal synthesis scheme for forming the blue color dispersion of $\mathrm{ReO}_{3} \mathrm{NCs}$ in chloroform (cuvette photograph on the right).

Although a favored route for metal oxide NC synthesis is through a nucleophilic "lysing" of the metal alkylcarboxylate complex, ${ }^{20}$ this strategy could not be employed in preparing $\mathrm{ReO}_{3} \mathrm{NCs}$. There are various reasons for this synthetic difficulty. The first one among them being the large number of available oxidation states for rhenium, which range from +3 to $+7 .{ }^{18}$ Controlling the oxidation state of rhenium then becomes challenging in the presence of long chain carboxylic acids, amines, or alcohols, ${ }^{14}$ the usual constituents of a general colloidal NC synthesis scheme. Furthermore, the unavailability of suitable precursors for the desired oxidation state and coordination number $(+6$, octahedral) makes it difficult to access the corresponding $\operatorname{Re}(+6)$-alkylcarboxylate, necessary for general metal oxide $\mathrm{NC}$ synthesis. The $\mathrm{Re}(+6)$-halides (like $\mathrm{ReF}_{6}, \mathrm{ReCl}_{6}$ ), which suit this purpose, are particularly reactive, and difficult to handle, and are also not readily available from commercial suppliers. They are extremely volatile and disintegrate upon heating at moderate temperatures, producing halogen gases. ${ }^{21}$

On the other hand, oxidation of a lower oxidation state rhenium precursor is hard to control as the reaction does not usually stop at the preferred +6 state $\left(\right.$ for $\mathrm{ReO}_{3}$ ) and goes on to form the +7 state. ${ }^{22}$ Bulk synthesis techniques have instead used a "soft" reduction of the +7 oxide $\left(\mathrm{Re}_{2} \mathrm{O}_{7}\right)$ with carbon monoxide $(\mathrm{CO})$ or ethers. ${ }^{22-23} \mathrm{We}$ chose ether-based reduction since the gaseous CO-reduction pathway is difficult to adapt in a liquid phase colloidal synthetic pathway. The nature of the reduction of $\operatorname{Re}(+7)$ is fundamentally different in these non-aqueous conditions, compared to typical aqueous redox conditions where reduction to $\mathrm{ReO}_{2}$ is more thermodynamically favorable than stopping the redox at $\mathrm{ReO}_{3},{ }^{24-25}$ and hence is suitable for our means. We adapted the ether based reaction in our synthesis scheme as that was demonstrated to be most successful for making bulk $\mathrm{ReO}_{3}$. In addition, long chain alcohols, amines, and alkylcarboxylic acids - all commonly employed as surfactants or reagents in colloidal NC synthesis - were found to be detrimental to $\mathrm{ReO}_{3} \mathrm{NC}$ synthesis as they consistently formed a brown amorphous product (Figure S3).

In our synthesis, the injection of $\operatorname{Re}_{2} \mathrm{O}_{7}$-TGY complex into a hot DOE-ODE mixture yielded the best results and the reaction temperature could be varied in a wide range owing to the high boiling points of both TGY $\left(275^{\circ} \mathrm{C}\right)$ and DOE $\left(286^{\circ} \mathrm{C}\right)$. ODE, being a noncoordinating solvent, enabled better control of the reaction kinetics. It lowers the concentration of the reducing agent (DOE), which leads to well-formed NCs and reduced aggregation in the as-synthesized products. Although $\mathrm{Re}_{2} \mathrm{O}_{7}$ is reduced by ethers, a molecule with multiple ether functionalities like TGY did not affect it in the reaction time frame. However, the $\mathrm{Re}_{2} \mathrm{O}_{7}$-TGY adduct should be freshly prepared as the solution turns brownish over time and cannot be stored (Figure S5). This suggests that TGY is sluggish in its reactivity towards $\mathrm{Re}_{2} \mathrm{O}_{7}$ and hence is an appropriate agent for delivering the 
$\mathrm{Re}_{2} \mathrm{O}_{7}$ into the reaction flask. The FTIR spectrum of a solution of $\mathrm{Re}_{2} \mathrm{O}_{7}$ in TGY exhibits a peak at around $909 \mathrm{~cm}^{-1}$ (Figure S1), which can be attributed to the Re-O-Re stretching mode of the $\mathrm{Re}_{2} \mathrm{O}_{7}$ molecule. ${ }^{26}$ A mixture of $\mathrm{Re}_{2} \mathrm{O}_{7}$ in DOE however turns green immediately at room temperature and signifies the high reactivity of $\mathrm{DOE}$ (Figure S5). This also signifies the suitability of DOE in our reaction as the reducing agent. Other ethers like dibenzyl ether, diphenyl ether, dioctadecyl ether, and dihexadecyl ether were also tested in this synthesis scheme. However, synthesis efforts with these molecules with aromatic functionalities or longer alkyl carbon chain did not yield the desired $\mathrm{ReO}_{3}$ phase, and instead led to the brown amorphous product described above.
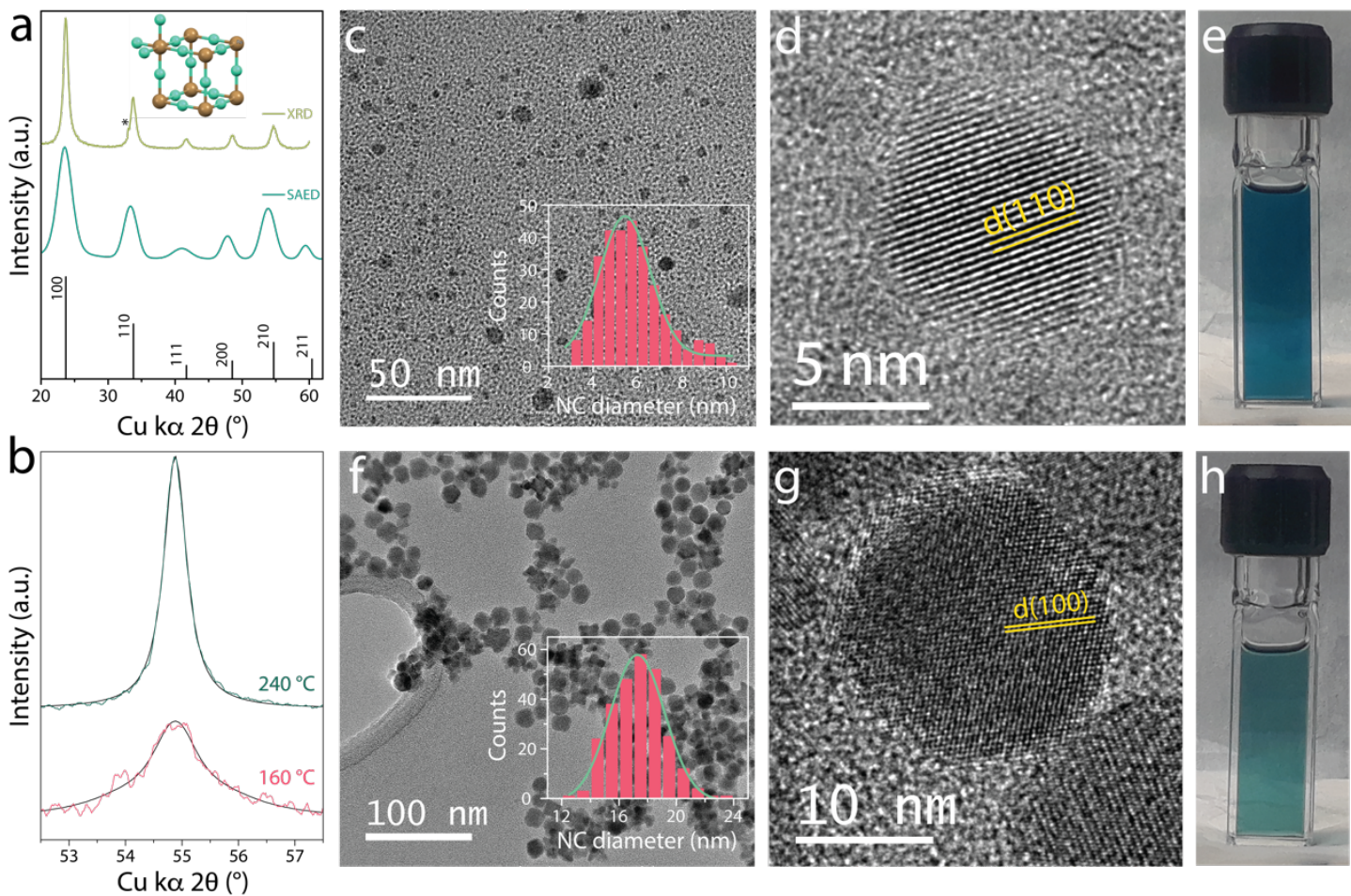

Figure 1. Structural and morphological characterization of the as-prepared $\mathrm{ReO}_{3} \mathrm{NCs}$. (a) XRD and azimuthally integrated SAED patterns, with reference to database powder XRD pattern ( $\mathrm{Pm} \overline{3} \mathrm{~m}, \mathrm{AMCSD} \# 0016967$ ). The unit cell of the $\mathrm{ReO}_{3}$ lattice is shown as well, with the Re atoms at the cube corners. (b) (210) XRD peak, along with corresponding fitted black curves for Scherrer analysis, for $\mathrm{ReO}_{3} \mathrm{NCs}$ obtained through low $\left(160{ }^{\circ} \mathrm{C}\right.$, red curve $)$ and high $\left(240^{\circ} \mathrm{C}\right.$, green curve $)$ temperature synthesis routes. TEM images (c,f) with size distribution histograms in the insets, and HRTEM images $(\mathrm{d}, \mathrm{g})$ of the NCs synthesized at $160^{\circ} \mathrm{C}(\mathrm{c}, \mathrm{d})$ and $240^{\circ} \mathrm{C}(\mathrm{f}, \mathrm{g})$. Colloidal stability of the as-synthesized NC solutions demonstrated by photographs of NCs in chloroform for the two synthesis routes (panels e $-160^{\circ} \mathrm{C} \& \mathrm{~h}-240^{\circ} \mathrm{C}$ ).

The as-synthesized $\mathrm{ReO}_{3} \mathrm{NCs}$ were then characterized for phase purity by XRD and azimuthally integrated SAED patterns (Figure 1a). The diffraction patterns match with the pattern for cubic $(\mathrm{Pm} \overline{3} \mathrm{~m})$ crystal structure of bulk $\mathrm{ReO}_{3}$ as reported by Meisel. ${ }^{27}$ Further evidence of phase purity is also offered by the NC Raman spectrum (Figure S2). Two different average sizes of the NCs were prepared at two different temperatures, 160 and $240{ }^{\circ} \mathrm{C}$. The $(210)$ peak for the two different sizes, along with their fitted pseudo-Voigt curves, are shown in Figure 1b. Scherrer analysis of these XRD peaks yield NC sizes of $7.6 \mathrm{~nm}\left(\mathrm{~T}=160^{\circ} \mathrm{C}\right)$ and $18.9 \mathrm{~nm}\left(\mathrm{~T}=240^{\circ} \mathrm{C}\right)$. These NC sizes were typical of those obtained for syntheses performed at two broad temperature ranges - temperatures below 200 ${ }^{\circ} \mathrm{C}$ yielded smaller NCs while larger NCs were produced when the reaction temperature was raised above $200{ }^{\circ} \mathrm{C}$. A representative TEM image for the smaller NCs is shown in Figure 1c with the size distribution histogram in the inset which yields an average size of 5.4 $( \pm 1.4) \mathrm{nm}$. On the other hand, Figure 1f shows a representative TEM image with accompanying size distribution histogram for the larger NCs with average size $17.3( \pm 1.9) \mathrm{nm}$. The sizes obtained from the XRD and TEM analyses were found to have a reasonable level of agreement - each being within the error margin of the other. HRTEM images of the smaller and larger NCs are respectively shown in Figures 1d and 1g, with the interplanar distances corresponding to different lattice planes of the $\mathrm{ReO}_{3}$ structure, signifying their crystalline nature. Each NC is a single crystal and no grain boundaries were observed within discrete NCs among those observed. The colloidal stability of these NCs is represented by their blue dispersions in chloroform (Figure 1e, h).

NC surface characteristics and solubility. The as-synthesized NC products and the reaction mixture were probed using FTIR and NMR analysis to gain further insight into the reaction and the NC surface characteristics. The as-synthesized NCs were found to be dispersible in chloroform. However, upon washing with an anti-solvent, the NCs tend to aggregate which signifies less than ideal coverage and bonding lability by organic ligands leading to reduced steric stabilization. Upon multiple purification steps, the organic moieties present on the NC surfaces completely disappear and the NCs exhibit better dispersibility in a polar solvent like DMF. A ${ }^{1} \mathrm{H}$ NMR spectrum of such a NC dispersion in deuterated DMF is included in Figure 2a, which shows a rather broad peak centered at around 4.2 ppm. The $2 \mathrm{D}^{1} \mathrm{H}-{ }^{13} \mathrm{C}$ correlation HSQC spectrum reveals that this signal is not associated with a corresponding carbon, although the DMF cross peaks can be identified (Figure 2a). Based on these observations, we assign the $4.2 \mathrm{ppm}$ broad signal to hydroxyl $(-\mathrm{OH})$ groups present on the NC surfaces. The broadening of the NMR signals indicate proximity to the NC surface, which causes magnetic inhomogeneities and restricted mobility of the proton leading to faster relaxation. ${ }^{28}$ This notion is further supported by the FTIR spectra 
shown in Figure $2 \mathrm{~b}$ : the washed NCs (blue curve) are devoid of any signals attributable to organic moieties, which were present in the unwashed NCs (black curve). A weak and relatively broad shoulder can be discerned around $3400 \mathrm{~cm}^{-1}$ which can be attributed to the surface hydroxyl groups. From these observations, we surmise that DOE acts as an L-type ligand (owing to the lone pair of electrons on the O-center), which imparts steric stabilization of the unwashed NCs in nonpolar solvents. However, each purification cycle leads to removal of these loosely bound molecules, leading to NC aggregation. In fact, this mixed ligand shell on the NC surface enables quick
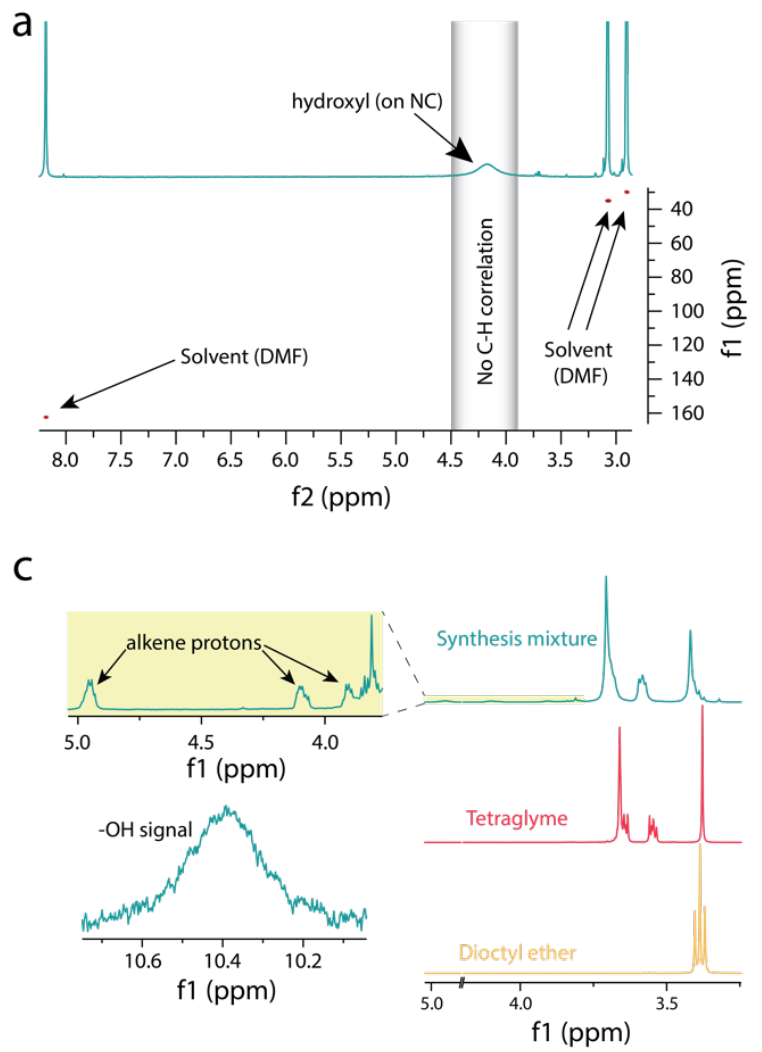

and easy switching from a nonpolar to a polar solvent. As shown in the inset of Figure $2 b$, the as-synthesized NCs can easily transfer from nonpolar hexane to polar DMF, by liberating the L-type DOE molecules in the hexane layer and without having to undergo a cumbersome ligand exchange process. This phase transfer with concomitant ligand stripping enables easy film processing from NC dispersions in polar solvents with limited contamination from organic ligands.
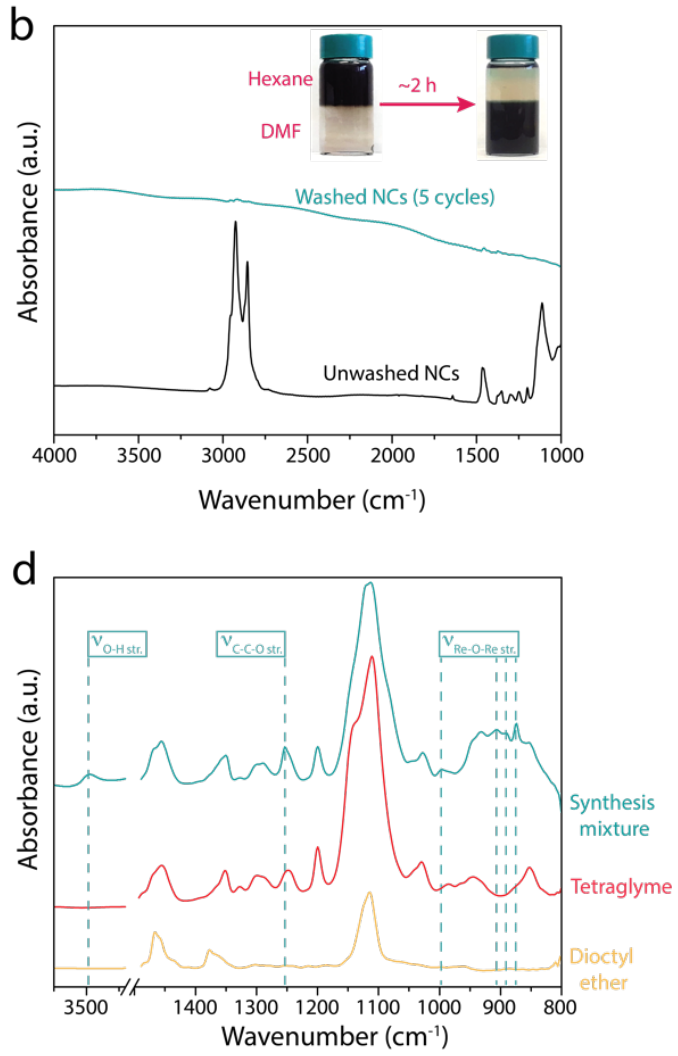

Figure 2. Surface chemistry of the as-prepared $\mathrm{ReO}_{3} \mathrm{NCs}$. (a) $1 \mathrm{D}{ }^{1} \mathrm{H}$ and $2 \mathrm{D}$ HSQC spectra for as-prepared $\mathrm{ReO}_{3} \mathrm{NCs}_{\mathrm{in}} \mathrm{DMF}_{7}$. No C-H crosspeaks were observed for the $4.2 \mathrm{ppm}$ peak, which was ascribed to surface-OH groups. (b) FTIR spectra of as-prepared $\mathrm{ReO}_{3} \mathrm{NCs}_{\text {before }}$ (black) and after 5 purification cycles (blue), showing washed NCs devoid of organic ligands. Inset shows the quick and easy transfer of the as-prepared NCs from the nonpolar hexane layer to the polar DMF layer owing to the mixed ligand shell. (c) $1 \mathrm{D}^{1} \mathrm{H}$ NMR spectrum, and (d) FTIR spectrum of the reaction mixture (blue curves), in comparison to the corresponding spectra of the constituents (TGY, red curves; and DOE, yellow curves). The hydroxyl signals were accompanied by alkene (in NMR, panel c) and enol (in FTIR, panel d) signals.

The presence of surface hydroxyl groups was further supported by XPS measurements of the as-synthesized $\mathrm{ReO}_{3} \mathrm{NCs}$. Air exposure was meticulously avoided before the measurement - the as-synthesized NCs were purified in a nitrogen-filled glove box, then transferred air-free to the XPS instrument. This procedure ruled out any post-synthetic changes that might occur on the NC surfaces due to air exposure. The Re 4f XPS (Figure S4a, blue curve), exhibits three peaks as opposed to a doublet ascribable to $\operatorname{Re} 4 f_{7 / 2}$ and $\operatorname{Re} 4 f_{5 / 2}$ for $\operatorname{Re}(+6)$. The reason for this deviation is the aforementioned large number of oxidation states for $\mathrm{Re}$, and their peak positions being within the $\mathrm{BE}$ range of $5-6 \mathrm{eV} \cdot{ }^{29}$ As a result, a sample might exhibit a triplet where the $\operatorname{Re} 4 f_{7 / 2}$ peak of a high oxidation state can overlap with the $\operatorname{Re} 4 \mathrm{f}_{5 / 2}$ peak of a lower oxidation state, given the small spinorbit splitting of $\operatorname{Re} 4 \mathrm{f}(2.42 \mathrm{eV})$ thus heavily convoluting the spectrum. As comprehensively explained by Greiner et al., ${ }^{29}$ this leads to the triplet for $\mathrm{ReO}_{3}$ samples when surface hydroxyls contribute to the signal thereby leading to an ill-defined oxidation state for surface $\operatorname{Re}$ atoms (a combination of +7 and +6 oxidation states). The $\operatorname{Re} 4 f$ spectrum for powder $\mathrm{Re}_{2} \mathrm{O}_{7}$ (orange curve) is also included in Figure S4a, which shows a well-defined doublet. The convoluted nature of the $\mathrm{Re} 4 \mathrm{fXPS}$ is directly related to the difference in the $\mathrm{O}$ 1s signals between $\mathrm{ReO}_{3} \mathrm{NCs}_{\text {and }} \mathrm{Re}_{2} \mathrm{O}_{7}$ powder (Figure S4b).

The presence of NC surface hydroxyl groups begets the questions of their origin, which required further investigation, especially since the presence of hydroxyl-generating moieties (moisture, for instance) was rigorously avoided, and the reactants used were aprotic. To this end, the synthesis mixture, immediately after cooling down post-injection, was probed using NMR and FTIR spectroscopy. The NMR spectrum of the synthesis mixture is shown in Figure 2c (blue curves), in comparison to that of TGY (red curve) and DOE (yellow curve). Additional peaks are discernible in the 3.8-5.0 ppm range (highlighted and blown-up region of the blue curve in Figure 2c) for the synthesis mixture, which are ascribable to alkene type protons (i.e. $\mathrm{C}=\mathrm{C}-\mathrm{H}$ groups). To rule out interference from alkene signals of ODE, this reaction was performed in its absence, i.e., only TGY and DOE were used. Furthermore, the formation of hydroxyl groups is 
also indicated by the broad signal at about $10.4 \mathrm{ppm}$ (Figure 2c) for the synthesis mixture. These results indicate that the surface hydroxyl groups are probably formed by hydrogen extraction from carbon chains present in the reaction mixture, given that only aprotic reactants have been employed in this synthesis. Considering that oxides of rhenium are used as catalysts in the dehydrogenation and reformation of hydrocarbons, ${ }^{29-30}$ this reaction mechanism is chemically plausible. A sketch of the likely reaction intermediate is included in Figure S6, which can be the precursor to surface hydroxyls. These NMR observations were complemented by FTIR measurements on the synthesis mixture, in comparison with that for TGY and DOE (Figure 2d). A relatively sharp hydroxyl -OH stretching mode, ascribable to molecular rather than NC surface hydroxyl signals, is discernible at about $3479 \mathrm{~cm}^{-1}$; while the C-C-O stretching mode due to an enol moiety is observed around $1253 \mathrm{~cm}^{-1}$. The enol moiety can form from the dehydrogenation of TGY, through the proposed reaction intermediate schematically shown in Figure S6. Since both TGY and DOE can serve as hydrogen donors essentially, it is not surprising to observe alkene and enol signature from the same reaction mixture. The rest of the weak signals $(874,891,998$ $\mathrm{cm}^{-1}$ ) can be ascribed to Re-O-Re bonds. ${ }^{26,31}$ In particular, the bands at 874 and $891 \mathrm{~cm}^{-1}$ can be ascribed to $\mathrm{Re}-\mathrm{OH}$ stretching, ${ }^{26}$ which lends further credence to the plausibility of the reaction scheme proposed. The wider range spectra for Figure $2 \mathrm{c}$ and $2 \mathrm{~d}$ are included in Figure S7.

In the above analysis, the NC surface chemistry was probed by characterizing the end and side products of the NC synthesis reaction. However, a full mechanistic elucidation of the NC synthesis will be required to gather a complete understanding of the pathway for surface $-\mathrm{OH}$ group formation. That will require an intensive spectroscopic investigation of the reaction intermediates, as has been recently done by Clark et al. for $\mathrm{Al} \mathrm{NCs.}{ }^{32}$ Though this goes beyond the scope of the present work, such mechanistic studies will be interesting in the future.

Optical extinction of the NCs- observation of LSPR. The UVVis extinction spectra of dilute dispersions of the as-prepared $\mathrm{ReO}_{3}$ NCs, synthesized at different temperatures are shown in Figure 3a. The spectra feature two absorption maxima at $410 \mathrm{~nm}(\approx 3 \mathrm{eV})$ and $590 \mathrm{~nm}(\approx 2.1 \mathrm{eV})$, and an "absorption-edge" type feature at wavelengths lower than $400 \mathrm{~nm}$ (energies greater than $3.1 \mathrm{eV}$ ). However, it is important to note at this point that even though $\mathrm{ReO}_{3}$ is an oxide, it actually is a metal and hence the conventional notion of a band-to-band transition in a semiconductor across a forbidden band gap does not describe the dominant light-matter interaction in this case. ${ }^{1-3}$ Hence, these extinction features are due to a combination of interband transitions and the characteristic LSPR bestowed due to nanostructuring. In the ensuing discussion, we show that the extinction maximum at $590 \mathrm{~nm}$ is indeed an LSPR response.

As reported by Feinleib et al., the dielectric function of $\mathrm{ReO}_{3}$ exhibits free electron behavior below the plasma edge of $2.1 \mathrm{eV}$, while interband transitions are the main optical mechanisms above this energy. ${ }^{1}$ The real and imaginary parts of the dielectric function for bulk $\mathrm{ReO}_{3}$ are shown in Figure S8a, where the real part of the dielectric function $\left(\varepsilon^{\prime}\right)$ passes the through zero at $2.18 \mathrm{eV}$ (the plasma energy or plasma frequency). Further, the imaginary part of the dielectric function $\left(\varepsilon^{\prime \prime}\right)$ is free electron-like up to $2.3 \mathrm{eV}$ with negligible value close to the plasma energy. This condition of a negative $\varepsilon^{\prime}$ accompanied by a negligible $\varepsilon^{\prime \prime}$ is the signature of a conductive material that can sustain an LSPR response. ${ }^{20,33}$ In addition, the peaks in the $\varepsilon^{\prime \prime}$ curve at energies higher than that correspond to interband transitions, which are associated with larger joint density of states at these energy levels of the electronic band structure. The dielectric function of $\mathrm{ReO}_{3}$ was deconvoluted into free- and bound-electron contributions, as the region of free electron behavior in $\mathrm{ReO}_{3}$ is delimited by the onset of interband transitions. A Drude treatment of the dielectric function leads to a plasma energy of $5.5 \mathrm{eV}$ (Figure $\mathrm{S} 8 \mathrm{~b}$ ), which, in the presence of the substantial bound electron contribution, leads to a zero crossing in the visible region $(2.18 \mathrm{eV}){ }^{1}$ This substantial influence of the bound-electron component on the free electron behavior in $\mathrm{ReO}_{3}$ is reminiscent of conventional metals like gold, silver, copper, and aluminum. ${ }^{34-36}$

Another quantity of relevance is the loss function - defined as $\operatorname{Im}\left(\varepsilon^{-1}\right)$ - wherein sharp maxima have been associated with plasma oscillations. ${ }^{1,34-35,37-39}$ The sharp peak in the loss function at the plasma energy (shown in Figure S9c) and associated small values of $\varepsilon^{\prime}$ and $\varepsilon^{\prime \prime}$, are indicative of the collective oscillations that can be expected in $\mathrm{ReO}_{3} \mathrm{NCs}$. These optical features support the observation of an LSPR response in nanocrystalline $\mathrm{ReO}_{3}$. On the other hand, the lowest energy interband transitions occurs between the oxygen $2 \mathrm{p}$ and the rhenium $5 \mathrm{~d}$-manifolds of $\mathrm{ReO}_{3}$ at about $3 \mathrm{eV},{ }^{1,5}$ and are consistent with the features present in the calculated bulk absorption coefficient ( $\alpha$; Figure S9c), which is equivalent to light absorption by a continuous slab of $\mathrm{ReO}_{3}$ and hence does not exhibit any features specifically ascribable to nanostructuring. ${ }^{33,40-41}$ The observation of the shoulder at $410 \mathrm{~nm}(\approx 3 \mathrm{eV})$ in Figure $3 \mathrm{a}$ is consistent with this notion. However, the optical characteristics of small particles deviate appreciably from those of the bulk solid, as a result of the surface mode excitations. ${ }^{33}$ This is especially true for small NCs made of a conductive material like $\mathrm{ReO}_{3}$, as can be appreciated from the appearance of the $590 \mathrm{~nm}(\approx 2.1 \mathrm{eV})$ band (Figure 3a) while there is no appreciable absorption feature in the $\alpha$ curve for the bulk material in that energy range (Figure S9c).

The absorption and scattering by small particles is best analyzed using the Mie solutions to Maxwell equations on electromagnetic waves interacting with a homogeneous sphere. ${ }^{33,42}$ The optical extinction of the $\mathrm{ReO}_{3} \mathrm{NCs}$ was quantitatively modeled using the quasi-static approximation of the Mie theory, which is applicable since the size of the NCs is much smaller than the wavelength of light (see section S9 for more details). The absorption cross-section $\sigma$ of a small nano-object per this theory is expressed as:

$$
\sigma_{A}=24 \pi^{2} R^{3} \sqrt{\varepsilon_{m}} / \lambda \frac{\varepsilon_{m} \varepsilon_{i}}{\left(\varepsilon_{r}+2 \varepsilon_{m}\right)^{2}+\varepsilon_{i}^{2}}
$$

where, $\varepsilon=\varepsilon_{r}+i \varepsilon_{i}$ is the frequency-dependent complex dielectric constant of the core material constituting the NC, while $\varepsilon_{m}$ is that of the medium in which the $\mathrm{NC}$ is immersed, $\mathrm{R}$ the $\mathrm{NC}$ radius, and $\lambda$ the wavelength. An enhancement in the absorption is observed when the denominator in eq 1 approaches zero - the socalled Fröhlich condition. ${ }^{43-44}$ The Fröhlich condition in metallic NCs is satisfied at wavelengths where the real part of the dielectric constant assumes a negative value, i.e., $\varepsilon_{r}=-2 \varepsilon_{m}$ and $\varepsilon_{i} \cong 0$. The presence of free charge carriers in metals facilitates the negative value of the real part of the dielectric constant, and specular reflectivity measurements on bulk $\mathrm{ReO}_{3}$ in air illustrate this same effect at energies below $2.18 \mathrm{eV}$ (the plasma edge). ${ }^{1}$ 

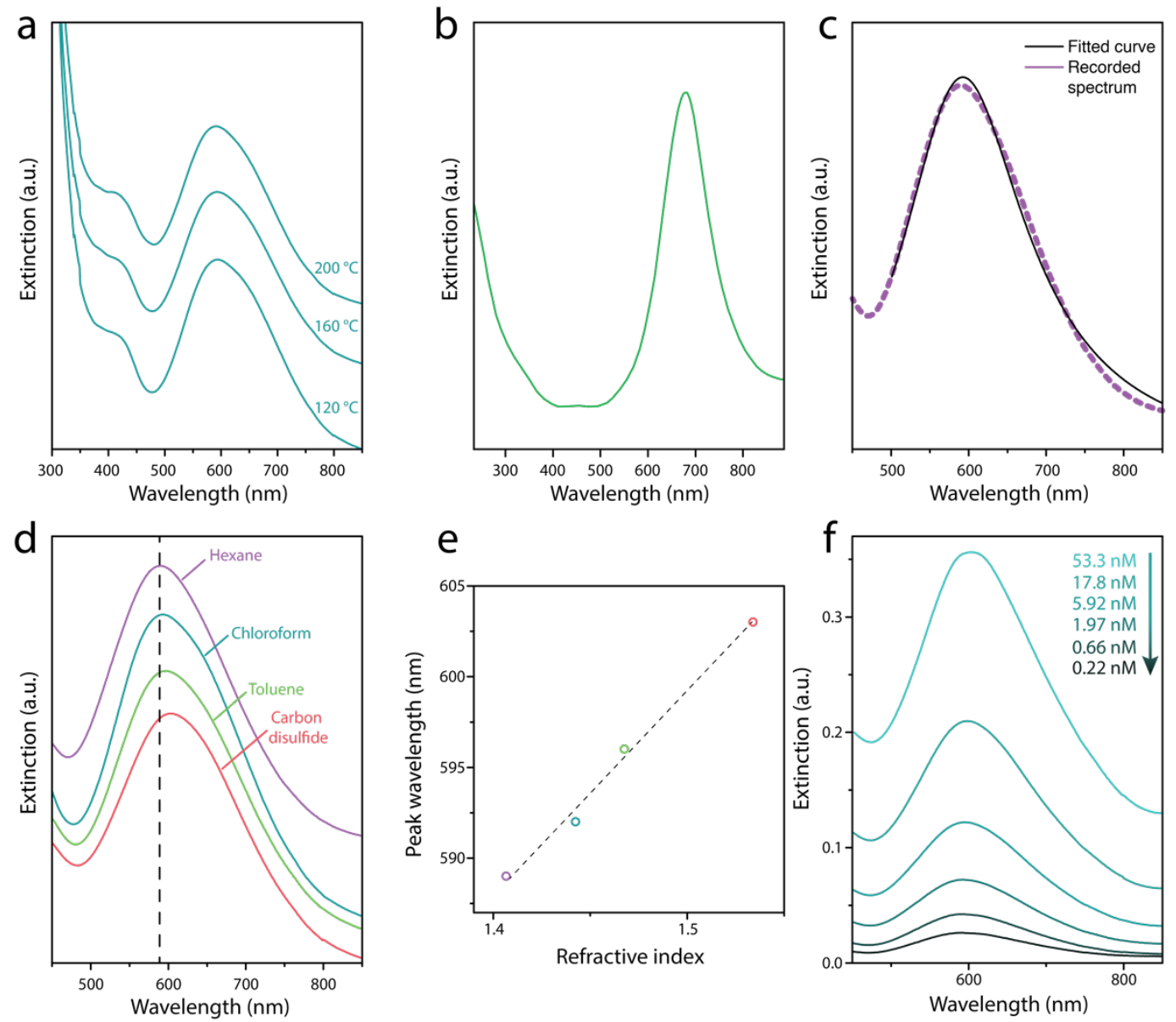

Figure 3. Optical extinction spectroscopy of the as-prepared $\mathrm{ReO}_{3} \mathrm{NCs}$ exhibiting LSPR response. (a) Spectra for as-synthesized NCs formed at different reaction temperatures $\left(<200^{\circ} \mathrm{C}\right)$ showing the LSPR band at around $590 \mathrm{~nm}$ and interband transition at around $410 \mathrm{~nm}$. (b) Spectrum calculated by Mie theory from the real and imaginary parts of the dielectric function of bulk $\mathrm{ReO}_{3}$. (c) Drude fit (black curve) to experimental spectrum (purple curve) of the NCs dispersed in hexane. (d) Spectra recorded in different solvents, showing the variation in the LSPR. (e) LSPR peak wavelength plotted as a function of the solvent refractive index, exhibiting a linear trend (fit shown). (f) Spectra recorded at different NC concentrations, for the calculation of molar attenuation coefficient (normalized curves shown in Figure S11).

The extinction spectrum calculated from the real and imaginary parts of the dielectric function of bulk $\mathrm{ReO}_{3}$, as per eq 1 , is shown in Figure $3 \mathrm{~b}$. The calculations were performed by the finite element method solutions to the Maxwell equations in the full field mode, and the details are included in section S9 with the model and geometry of the simulations shown in Figure S10. The prominent peak in the spectrum is ascribed to the LSPR response of these NCs, as no such peak appears in the $\alpha$ curve (bulk absorption coefficient; Figure S9c). Considering that the collective oscillations of the free electrons present in metallic $\mathrm{ReO}_{3} \mathrm{NCs}$ are responsible for this LSPR response, the optical modes experimentally observed in the 500-850 $\mathrm{nm}$ range were fit using the Drude model (section S10). The Drude contribution to the dielectric function $\left(\varepsilon_{D}(\omega)\right)$ is expressed as:

$$
\varepsilon_{D}(\omega)=\varepsilon_{\infty}-\frac{\omega_{p}^{2}}{\omega^{2}+i \omega \Gamma}
$$

where, $\varepsilon_{\infty}$ is the high-frequency dielectric constant $\left(1\right.$, for $\mathrm{ReO}_{3}$, Figure S8). The bulk plasma frequency $\left(\omega_{p}\right)$ is expressed as a function of the free electron density $(n)$, electronic charge $(e)$, permittivity of vacuum $\left(\varepsilon_{0}\right)$ and electron effective mass: $\left(\omega_{p}^{2}=\right.$ $\left.n e^{2} / \varepsilon_{0} m^{*}\right)$. $\Gamma$ is the damping constant and is a measure of electron scattering in the bulk and at the NC surfaces. The Drude fit to the optical extinction spectrum for $\mathrm{ReO}_{3} \mathrm{NCs}$ in hexane (Figure 3c) enabled us to extract quantitative information. The plasma frequency was calculated to be between $4.7-4.9 \mathrm{eV}$, depending on the solvent used in the spectral acquisition (section S10), which is close to the calculated $5.5 \mathrm{eV}$ by Feinleib et al. ${ }^{1}$ The electron concentrations obtained were in the range of $1.58-1.75 \times 10^{22} \mathrm{~cm}^{-3}$, which are close to the theoretical value of $1.78 \times 10^{22} \mathrm{~cm}^{-3}$ considering one electron per $\mathrm{ReO}_{3}$ unit.

Very similar spectra were obtained for reactions performed at temperatures below $200{ }^{\circ} \mathrm{C}$ (Figure 3a), which suggested similar sizes for the NCs. As described above, only at reaction temperatures above $200^{\circ} \mathrm{C}$, we observed the formation of NCs with larger diameters. However, reactions at those temperatures also led to increased aggregation (Figure 1f), which can be explained by reduced ligand coverage, and increased coalescence of the growing NCs. The optical extinction spectrum for NCs synthesized at these higher temperatures feature line broadening (Figure S11) due to increased interNC plasmonic coupling that gives rise to a red shift and potentially increased scattering. ${ }^{45}$ In fact, when the NCs synthesized at lower temperature lose their L-type ligand shells upon multiple washings, they exhibit similar spectral characteristics. Analogous spectral changes are observed when NCs are dropcast on glass substrates 
(Figure S11). These observations provide further evidence that the absorption band at $590 \mathrm{~nm}$ in the NC dispersions is an LSPR response.

The LSPR response observed at $590 \mathrm{~nm}$ is sensitive to the surrounding medium, i.e., the solvent in which these NCs are immersed (Figure 3d). In particular, the LSPR peak position linearly redshifts as the refractive index of the solvent increases (Figure 3e). This is consistent with the expected behavior of an LSPR response, as has been demonstrated for various other metal nanoparticles and doped semiconductor NCs. ${ }^{45-46}$ The sensitivity factor $\left(S_{\mathrm{F}}\right)$, defined as the shift of the LSPR peak wavelength per unit change in refractive index of the surrounding medium, was determined from the slope of the linear fit shown in Figure 3e. For $\mathrm{ReO}_{3} \mathrm{NCs}$, the $\mathrm{S}_{\mathrm{F}}$ was $112.3( \pm$ 6.8), which compares well with values for similar sized $\mathrm{Au}(44 \pm 3),{ }^{47}$ and Ag NCs (87). ${ }^{48}$ Similar sensitivity has also been observed in NCs of $\mathrm{LaB}_{6}$, which has similar origins of metallic behavior as $\mathrm{ReO}_{3}$ (i.e. one extra La-electron per $\mathrm{LaB}_{6}$ unit). ${ }^{49}$

The molar attenuation coefficient $(\epsilon)$ of these $\mathrm{ReO}_{3} \mathrm{NCs}$ was determined from a dilution series and using the Lambert-Beer law. Figure $3 \mathrm{f}$ shows the six spectra with the respective NC concentrations that constitute the dilution series. The $\mathrm{NC}$ concentration for the mother solution was determined from the Re-content by elemental analysis using ICP-OES, considering the NC size from TEM imaging described above. As per the Lambert-Beer law, absorbance at a certain wavelength $(A)$ can be expressed as the function of $\epsilon$, optical path length $(b)$ in $\mathrm{cm}$, and the molar concentration of the NCs (c) i.e. $A=\epsilon \times b \times c$. The value of the $\epsilon$ for $\mathrm{ReO}_{3} \mathrm{NCs}$ obtained by this method was $3.3 \times 10^{7} \mathrm{M}^{-1} \mathrm{~cm}^{-1}$ at the LSPR peak, and is comparable to that for similar sized $\mathrm{Au} \mathrm{NCs}^{50-51}$ and an order of magnitude smaller than that for $\mathrm{Ag} \mathrm{NCs,}{ }^{52-53}$ at their respective LSPR peaks at visible wavelengths.

Optical modulation of NC films. EC ion (de)insertion can induce reversible optical modulation in degenerately doped metal oxide NCs. ${ }^{54-58}$ We sought to investigate the effects of such ion (de)insertion on the spectral response of the inherently metallic $\mathrm{ReO}_{3} \mathrm{NC}$ films under EC biasing. The NC films were used as the working electrode in these experiments while the optical extinction of the films was recorded in situ (Figure 4). The EC (dis)charging was carried out with two different cations $-\mathrm{Li}^{+}$and the bulky $\mathrm{TBA}^{+}$ions. The choice of the cations for these measurements was driven by the fact that $\mathrm{Li}$-ions are inserted into a host oxide lattice more readily while that effect can be essentially inhibited using the bulky TBA-ions. ${ }^{59}$

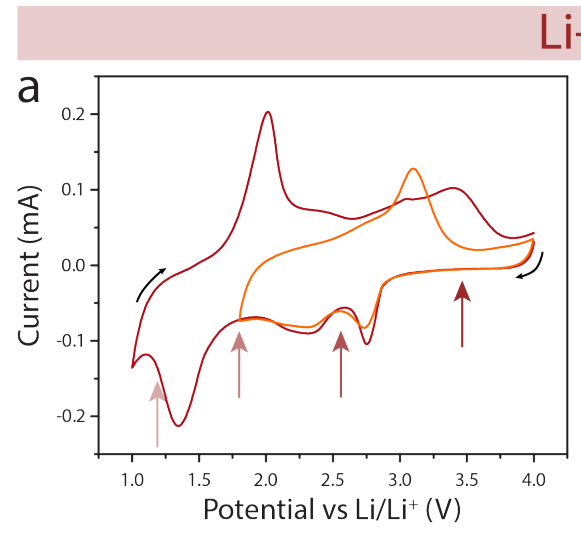

Li-ion cycling
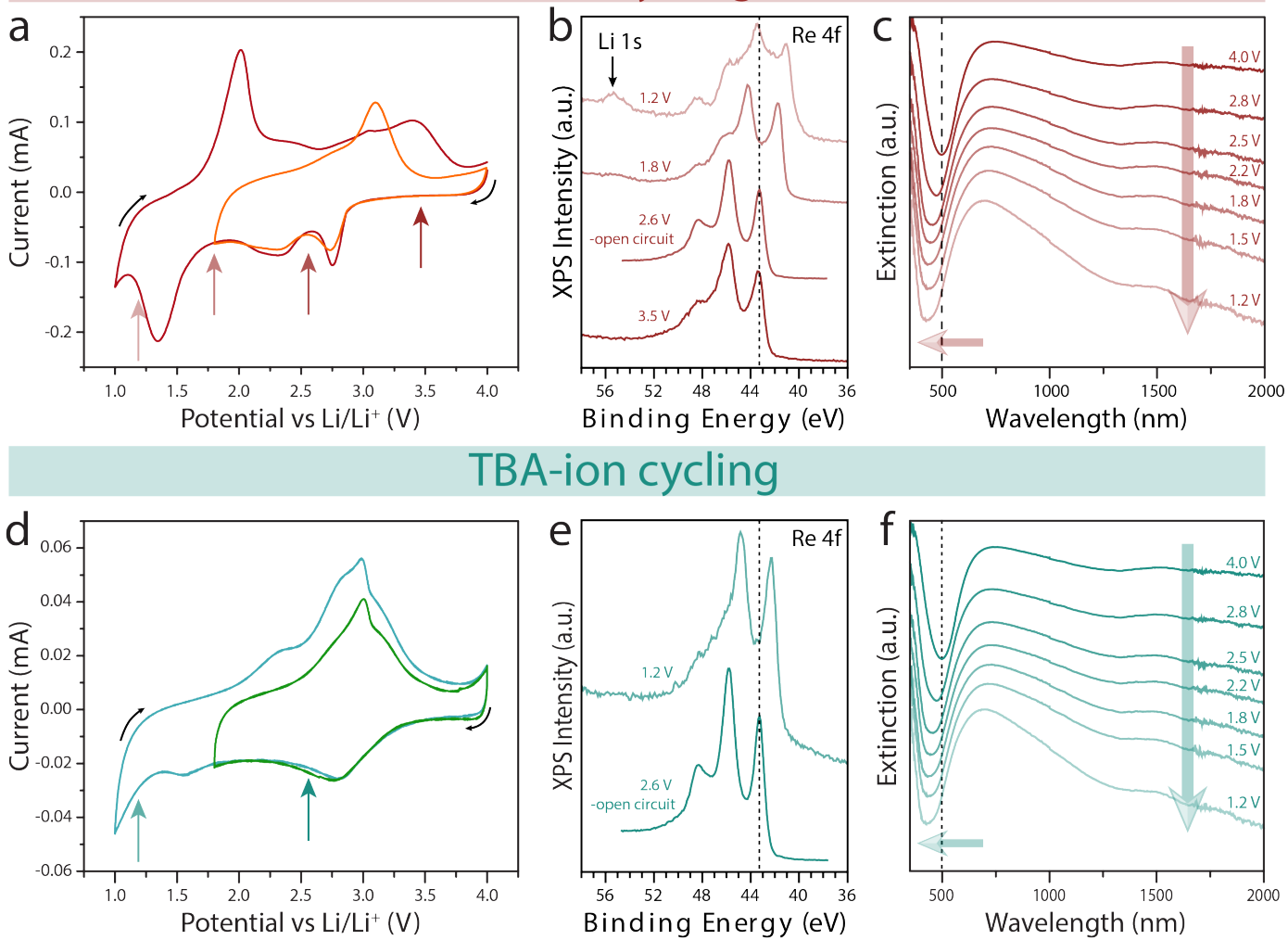

BA-ion cycling
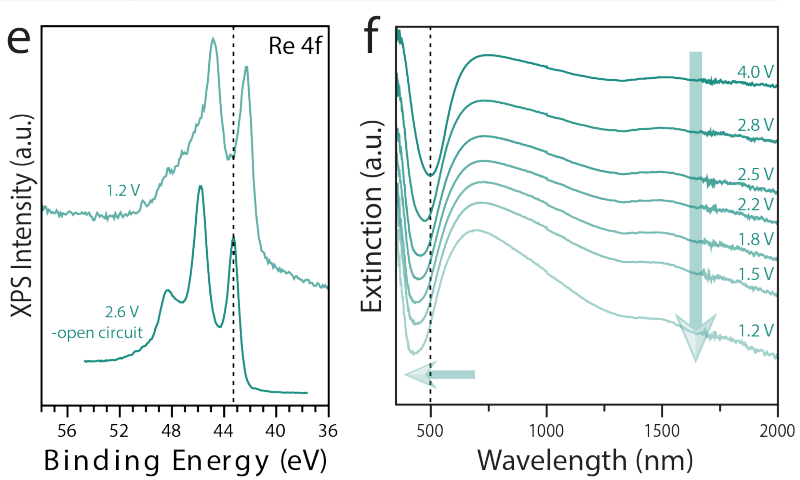

Figure 4. EC (dis)charging and optical modulation of dropcast $\mathrm{ReO}_{3} \mathrm{NC}$ films for Li-ion (a-c) and TBA-ion (d-f) cycling. (a, d) CV scans, (b, e) ex situ high-resolution XPS scans of the Re $4 \mathrm{f}$ regions, and (c, f) in situ optical extinction spectra for the NC films charged to different potentials. (a) Liion CV scans for potential ranges: $1.8-4 \mathrm{~V}$ (orange curve; reversible), and $1-4 \mathrm{~V}$ (red curve, irreversible). (d) TBA-ion CV scans at potential ranges: $1.8-4 \mathrm{~V}$ (green curve; reversible), and $1-4 \mathrm{~V}$ (blue curve; reversible). Arrow markers in CV scans in panel a (d) correspond to the respective potentials at which the XPS spectra in panel $b(e)$ were recorded. The XPS scan for the as-synthesized NCs is denoted as open circuit. In panels $c$ and $f$, the arrow markers signify loss of NIR intensity and blue shift of the LSPR-interband valley, as the films are charged.

The CV scans for the Li-ion and TBA-ion (dis)charging cycles are shown in Figures 4a and d, respectively. In a charging cycle, the progression of potentials was from $4 \mathrm{~V}$ to $1 \mathrm{~V}$ for injecting electrons into the NC films. The Li CV scans (Figure 4a) exhibit two distinct sets of redox peaks - at 2.75 and $1.3 \mathrm{~V}$ in the charging (or reducing) trace, consistent with the recent bulk EC studies. ${ }^{60}$ Similar redox sets were also observed in the TBA CV scans albeit with almost non-existent $1.3 \mathrm{~V}$ peak (Figure $4 \mathrm{~d}$ ). However, there are several differences between the nature of the EC cycling with the two ions. The first is that of reversibility - in the measured potential range of $1-4 \mathrm{~V}$, the reversible (de)insertion of $\mathrm{Li}$ is compromised once the films are 
charged to potentials lower than $1.5 \mathrm{~V}$, i.e., once the charging proceeds beyond the second redox peak at $1.3 \mathrm{~V}$. The material breaks down at these strong reducing potentials, as evidenced by the emergence of multiple peaks in the subsequent oxidizing trace at potentials above $2.5 \mathrm{~V}$. The breakdown apparently occurs even in the first scan and subsequent scans exhibit a capacitive nature of charging with no distinct redox peaks (Figure S12). The Li CV scans cycle reversibly in the $1.8-4 \mathrm{~V}$ range, however. On the contrary, the TBA $\mathrm{CV}$-scans remain reversible in a larger potential range of $1-4 \mathrm{~V}$ (Figure S13).

Another major difference in the CV scans is the magnitude of the current recorded for the same scan rate $(10 \mathrm{mV} / \mathrm{s})$ and similar film surface area and thickness for each ion. The currents for the TBAion are about an order of magnitude lower than that recorded for the $\mathrm{Li}$-ion charging. Furthermore, the pronounced peak at $1.3 \mathrm{~V}$ for $\mathrm{Li}$ scan is of negligible magnitude in the TBA-scan (compare Figures $4 \mathrm{a}$ and $\mathrm{d}$ ). Based on prior literature, the observations made for the $\mathrm{Li}$ ion scans can be correlated with the progressive insertion of $\mathrm{Li}$ ions in the $\mathrm{ReO}_{3}$ lattice accompanied by transformations to multiple phases generally formulated as $\mathrm{Li}_{x} \mathrm{ReO}_{3}{ }^{60}$ In fact, the $\mathrm{ReO}_{3}$ lattice can accommodate up to two $\mathrm{Li}$ ions per unit of $\mathrm{ReO}_{3}$ (i.e., $\mathrm{x}=2$ ), leading to the $\mathrm{Li}_{2} \mathrm{ReO}_{3}$ phase. ${ }^{61}$ This is feasible due to the openframework of the lattice (Figure S14) which arises from the missing large central A-site cation in this perovskite structure. Accommodating one $\mathrm{Li}$ ion in each $\mathrm{A}$-site (forming $\mathrm{LiReO}_{3}$ ) involves little structural reorganization and can be done reversibly, consistent with the reversibility illustrated in the $1.8-4 \mathrm{~V}$ range (Figure S12). However, the A-site requires significant expansion when more than one $\mathrm{Li}$ ion occupies the same site, as will be the case on average for $\mathrm{x}>1$. This leads to significant structural distortion involving rotations of the $\mathrm{ReO}_{6}$ octahedra (Figure S14), which eventually blocks the easy Liion transport previously possible in the highly symmetric cubic structure. ${ }^{60}$ This regime is signified by the $1.3 \mathrm{~V}$ reduction peak (Figure 4a) and also explains the irreversibility of the $\mathrm{Li}(\mathrm{de})$ insertion after the first scan (Figure S12). These conclusions are corroborated by ex situ XPS in the Re 4f range (Figure 4b). As discussed previously in the context of Figure S4, the Re $4 \mathrm{f}$ signal for the as-synthesized NCs is highly convoluted and exhibits a triplet instead of a doublet. These peaks progressively shift to lower values of $\mathrm{BE}$ as the extent of $\mathrm{Li}$ incorporation increases, signifying reduction in the Re oxidation state and presumed formation of $\mathrm{LiReO}_{3}$ (at $1.8 \mathrm{~V}$ ) and $\mathrm{Li}_{(1+\mathrm{x})} \mathrm{ReO}_{3}($ at $1.2 \mathrm{~V}) .^{60}$ The incorporation of $\mathrm{Li}$ is also evident from the appearance of the Li 1s signal in XPS (marked for the $1.2 \mathrm{~V} \mathrm{spec-}$ trum). $\mathrm{Li}$ can reduce $\mathrm{ReO}_{3}$ even further as demonstrated by the further shift in the Re 4f XPS of the NCs deposited on a cleaned Li foil, compared to the $1.2 \mathrm{~V}$ spectrum (Figure S15). The fact that we do not observe such level of reduction in our EC experiments suggests that the structure disintegration occurs much before the limit of $2 \mathrm{Li}$ ions per $\mathrm{ReO}_{3}$ unit is reached.

Owing to the relative bulk of the TBA-ion inhibiting lattice ion insertion, we do not expect to observe distinct redox peaks in the CV scans involving this ion and (dis)charging is expected to be primarily of a capacitive nature. However, the prominent $2.75 \mathrm{~V}$ reduction peak (Figure 4d) can be ascribed to the small size of the NCs, which allows for a substantial current due to pseudocapacitive effects. Open surface A-sites can conceivably accommodate TBA ions that charge compensate injected electrons that reduce the Re ions. The lack of long alkyl chains on the washed NC surfaces, as discussed above, can facilitate the approach and surface interaction of TBA ions. The relatively small currents in the CV scans using TBA support this interpretation, as does the absence of a second redox peak at $1.3 \mathrm{~V}$ (as observed for $\mathrm{Li}$ ) since it is likely infeasible to accommodate two bulky TBA ions on an A-site, even one located at the NCelectrolyte interface. Pseudocapacitive charging has been demonstrated to contribute significantly in other nanostructured metal oxides. ${ }^{62}$ EX situ XPS measurements following reduction in a TBA electrolyte (Figure 4f) indicate reduction of the Re albeit to a lesser degree than with $\mathrm{Li}$, as the shifts of the $\operatorname{Re} 4 \mathrm{fBE}$ are lower in magnitude when comparing reduction at $1.2 \mathrm{~V}$ between the two ions (compare Figures $4 \mathrm{e}$ and $\mathrm{f}$ ).

The in situ recorded optical extinction spectra for these ion (de)insertion experiments exhibited changes that are consistent with previously reported electrochemically induced phase transformations of $\mathrm{ReO}_{3}$ lacking a distinct nanostructure. The spectral changes are strikingly similar for charging with Li (Figure 4c) or TBA (Figure 4f), suggesting similar underlying mechanisms. The films were charged back to the open circuit potential after each spectral acquisition at a particular potential, in order to ensure reversibility. The TBA spectra could be recovered to the open circuit spectrum after (dis)charging to each potential shown in Figure 4f, signifying the reversibility of the process over the entire potential range. However, the Li spectra could not be recovered to the open circuit spectrum once the film was charged to potentials lower than $1.8 \mathrm{~V}$, consistent with the irreversibility of EC cycling beyond that potential with a Li electrolyte. The extinction in the near-infrared (NIR) range declines as the films are progressively charged, accompanied by a blue shift of the valley between the LSPR and interband transition. The valley moves from about $500(2.48 \mathrm{eV}) \mathrm{nm}$ to $435 \mathrm{~nm}$ $(2.85 \mathrm{eV})$ regardless of the cation in the electrolyte.

These results can be understood by the previously reported EC phase transformations associated with the lithium (de)insertion in $\mathrm{ReO}_{3}$. $\mathrm{Li}_{\mathrm{x}} \mathrm{ReO}_{3}$ remains metallic for most values of $\mathrm{x}$ up to $\mathrm{x}=2,{ }^{60}$ so an LSPR response is expected in our NC films even as they progress through various states of charge. In fact, the broad extinction peak that we ascribe to LSPR is observed at all potentials (Figure S11b). However, different metallic phases are expected to exhibit distinct plasma energies owing to varying free electron densities and electronic band structures, leading to changes in plasma energies and effective masses. These changes are expected to influence the LSPR peak position and intensity of absorption in the NIR, consistent with the observed blue shifts in the LSPR-interband valley and increased extinction in the NIR at reducing potentials. The spectroscopic position of interband absorption features is also expected to change due to changes in the electronic band structure. Hence, the spectral responses recorded in Figure $4 \mathrm{e}$ are understood as the combined effect of all these mechanisms. Similar conclusions can also be drawn in case of TBA cycling in Figure 4f, although TBA cycling has the added advantage of being reversible. This elucidated plasmonic modulation in metallic $\mathrm{ReO}_{3} \mathrm{NCs}$ differs fundamentally from traditional metallic systems like Ag or Au NCs where capacitive charging is the only significant EC modulation mechanism. ${ }^{63-64}$

Since polaronic effects during $\mathrm{Li}$ insertion in bulk $\mathrm{ReO}_{3}$ are excluded, ${ }^{60}$ this material in its bulk form has so far been considered uninteresting from the point of view of an electrochromic oxide. ${ }^{65}$ However, obtaining colloidal NCs of this material has enabled the observation of LSPR and consequently the interesting SEC responses described here. The ability to synthesize discrete NCs and process these into films should enable a wide array of fundamental investigations and potential applications.

\section{CONCLUSIONS}

We have described a hot-injection synthesis procedure for col- 
loidal $\mathrm{ReO}_{3} \mathrm{NCs}$, which progresses through an ether-based reduction. This intriguing metallic system has not been previously synthesized by colloidal chemistry and our success at this has enabled the first fundamental studies of LSPR phenomena emerging in nanoscale $\mathrm{ReO}_{3}$. This material is remarkable in that it is an oxide and yet exhibits significant free electron density owing to the one conduction band electron per $\mathrm{ReO}_{3}$ unit. Motivated by the complicated redox chemistry of rhenium, the chemical synthesis itself is a departure from the usual methods associated with making colloidal metal oxide NCs - it does not proceed through the more common metal alkylcarboxylate nucleophilic lysis where the oxidation state of the metal cation remains constant throughout. Instead, a "soft" reduction of rhenium (+7) oxide in organic media containing long carbon chain ethers was used to produce $\mathrm{ReO}_{3} \mathrm{NCs}$. The ether molecules bind to the NC surface, and so do hydroxyl moieties generated during the reaction. This mixed ligand shell imparts an unusual flexibility in switching between nonpolar and polar solvents, which facilitates easy processing, e.g., into electrochemically active films as demonstrated here.

The colloidal stability of the $\mathrm{ReO}_{3} \mathrm{NC}$ dispersions enabled us to investigate the optical extinction of these plasmonic NCs and facilitated our rationalization of their optical spectra as a combination of interband and LSPR contributions, based on interpretation supported by Mie theory calculations and Drude fitting. The broadened LSPR response in the NC films enabled the observation of optical modulation in these films under EC biasing with Li and TBA ions. Such optical modulation was hitherto not observed in bulk $\mathrm{ReO}_{3}$ although reversible phase transformations to different metallic phases accompanying these ion (de)insertion experiments have been reported earlier for bulk $\mathrm{ReO}_{3}$. This optical modulation serves as an example of the advantages of producing $\mathrm{ReO}_{3}$ in the nanoscale. Considering that this synthesis method can produce large quantities of these NCs fairly quickly, it should inspire further investigations of these NCs such as in molecular sensing and plasmonic photocatalysis.

\section{ASSOCIATED CONTENT}

Supporting Information. FTIR spectrum and photographs of $\mathrm{Re}_{2} \mathrm{O}_{7^{-}}$ tetraglyme complex and $\mathrm{Re}_{2} \mathrm{O}_{7}$ in dioctyl ether; Raman and XPS spectra of $\mathrm{ReO}_{3} \mathrm{NCs}$; XRD and photograph of the brown amorphous product; scheme of the proposed reaction intermediate; NMR and FTIR spectra of the synthesis mixture; optical constants of bulk $\mathrm{ReO}_{3}$; COMSOL simulations of NC optical extinction spectrum; Drude modeling of LSPR response; effects of aggregation on extinction spectrum; reversibility of $\mathrm{CV}$ curves with $\mathrm{Li}$ and TBA ions; crystal structure of $\mathrm{ReO}_{3}$; High-resolution XPS of $\mathrm{ReO}_{3} \mathrm{NCs}$ on a Li-foil.

\section{AUTHOR INFORMATION}

\section{Corresponding Author}

*E-mail: milliron@che.utexas.edu

\section{ACKNOWLEDGMENTS}

This work was funded by the National Science Foundation (CHE1609656, CBET-1704634, and the Center for Dynamics and Control of Materials MRSEC, DMR-1720595), the Welch Foundation (F-1848), and the Fulbright Program (IIE-15151071). Support for H.-C. L. is acknowledged from CBMM. Helpful discussions with Sungyeon Heo regarding SEC are acknowledged. Useful discussion with Matthew Sheldon of Texas A\&M University about electromagnetic simulations of NC extinction is acknowledged. The authors acknowledge the timely help extended by various user facility staff for the measurements and data analysis: Karalee Jarvis of Texas Materials Institute (TMI) for electron microscopy; Hugo Celio of TMI for XPS measurements, and Steven D. Sorey and Garrett Blake of the UT Austin NMR facility for NMR measurements. Marvin was used for drawing, displaying and characterizing chemical structures, substructures and reactions, Marvin 17.21.0, ChemAxon (https://www.chemaxon.com).

\section{REFERENCES}

(1) Feinleib, J.; Scouler, W. J.; Ferretti, A., Optical Properties of the Metal $\mathrm{ReO}_{3}$ from 0.1 to $22 \mathrm{eV}$. Phys. Rev. 1968, 165, 765-774.

(2) Ferretti, A.; Rogers, D. B.; Goodenough, J. B., The relation of the electrical conductivity in single crystals of rhenium trioxide to the conductivities of $\mathrm{Sr}_{2} \mathrm{MgReO}_{6}$ and $\mathrm{Na}_{\mathbf{x}} \mathrm{WO}_{3}$. J. Phys. Chem. Solids 1965, 26, 2007-2011.

(3) Pearsall, T. P.; Lee, C. A., Electronic transport in $\mathrm{ReO}_{3}$ : dc conductivity and Hall effect. Phys. Rev. B 1974, 10, 2190-2194.

(4) Allen, P. B.; Schulz, W. W., Bloch-Boltzmann analysis of electrical transport in intermetallic compounds: $\mathrm{ReO}_{3}, \mathrm{BaPbO}_{3}, \mathrm{CoSi}_{2}$, and $\mathrm{Pd}_{2} \mathrm{Si}$. Phys. Rev. B 1993, 47, 14434-14439.

(5) Mattheiss, L. F., Band Structure and Fermi Surface of ReO3. Phys. Rev. 1969, 181, 987-1000.

(6) Mattheiss, L. F., Crystal-Field Effects in the Tight-Binding Approximation: $\mathrm{ReO}_{3}$ and Perovskite Structures. Phys. Rev. B 1970, 2, 3918-3935.

(7) Wertheim, G. K.; Hüfner, S., X-Ray Photoemission Band Structure of Some Transition-Metal Oxides. Phys. Rev. Lett. 1972, 28, 1028-1031.

(8) Narath, A.; Barham, D. C., Nuclear Magnetic Resonance in the Metal $\mathrm{ReO}_{3}$ : ${ }^{185}$ Re and ${ }^{187}$ Re Knight Shifts and Spin Relaxation Rates. Phys. Rev. 1968, 176 479-483.

(9) Pearsall, T. P.; Coldren, L. A., Stiffness matrix and debye temperature of $\mathrm{ReO}_{3}$ from ultrasonic measurements. Solid State Commun. 1976, 18, 1093-1096. (10) Biswas, K.; Rao, C. N. R., Metallic $\mathrm{ReO}_{3}$ Nanoparticles. J. Phys. Chem. B 2006, 110, 842-845.

(11) Chong, Y. Y.; Fan, W. Y., Facile Synthesis of Single Crystalline Rhenium (VI) Trioxide Nanocubes with High Catalytic Efficiency for Photodegradation of Methyl Orange. J. Colloid Interface Sci. 2013, 397, 18-23.

(12) Ghosh, S.; Biswas, K.; Rao, C. N. R., Core-shell nanoparticles based on an oxide metal: $\mathrm{ReO}_{3} @ \mathrm{Au}(\mathrm{Ag})$ and $\mathrm{ReO}_{3} @ \mathrm{SiO}_{2}\left(\mathrm{TiO}_{2}\right)$.J. Mater. Chem. 2007, 17, 2412-2417.

(13) Maitra, U.; Ghosh, S.; Biswas, K.; Rao, C. N. R., Scaling behavior of plasmon coupling in $\mathrm{Au}$ and $\mathrm{ReO}_{3}$ nanoparticles incorporated in polymer matrices. Phys. Status Solidi RRL 2010, 4, 169-171.

(14) Jeong, Y.-K.; Lee, Y. M.; Yun, J.; Mazur, T.; Kim, M.; Kim, Y. J.; Dygas, M.; Choi, S. H.; Kim, K. S.; Kwon, O.-H.; Yoon, S. M.; Grzybowski, B. A., Tunable Photoluminescence across the Visible Spectrum and Photocatalytic Activity of Mixed-Valence Rhenium Oxide Nanoparticles. J. Am. Chem. Soc. 2017, 139, 15088-15093.

(15) Dong, A.; Ye, X.; Chen, J.; Kang, Y.; Gordon, T.; Kikkawa, J. M.; Murray, C. B., A Generalized Ligand-Exchange Strategy Enabling Sequential Surface Functionalization of Colloidal Nanocrystals. J. Am. Chem. Soc. 2011, 133, 9981006.

(16) Langford, J. I.; Wilson, A. J. C., Scherrer after sixty years: A survey and some new results in the determination of crystallite size. J. Appl. Crystallogr. 1978, 11, 102-113.

(17) Gammer, C.; Mangler, C.; Rentenberger, C.; Karnthaler, H. P., Quantitative local profile analysis of nanomaterials by electron diffraction. Scr. Mater. 2010, 63, 312-315.

(18) Greenwood, N. N.; Earnshaw, A., 24 - Manganese, Technetium and Rhenium. In Chemistry of the Elements (Second Edition), ButterworthHeinemann: Oxford, 1997; pp 1040-1069.

(19) Cotton, F. A.; Wilkinson, G., Advanced inorganic chemistry. John Wiley \& Sons, Incorporated: 1988.

(20) Agrawal, A.; Cho, S. H.; Zandi, O.; Ghosh, S.; Johns, R. W.; Milliron, D. J., Localized Surface Plasmon Resonance in Semiconductor Nanocrystals. Chem. Rev. 2018, 118, 3121-3207.

(21) Peacock, R. D., 39 - RHENIUM. In The Chemistry of Manganese, Technetium and Rhenium, Pergamon: 1973; pp 905-978.

(22) Nechamkin, H.; Hiskey, C. F.; Moeller, T.; Shoemaker, C. E., Rhenium(VI) Oxide (Rhenium Trioxide). In Inorg. Synth., John Wiley \& Sons, Inc.: 2007; pp 186-188. 
(23) Nechamkin, H.; Kurtz, A. N.; Hiskey, C. F., A Method for the Preparation of Rhenium (VI) Oxide. J. Am. Chem. Soc. 1951, 73, 2828-2831.

(24) King, J. P.; Cobble, J. W., Thermodynamic Properties of Technetium and Rhenium Compounds. VI. The Potential of the $\mathrm{ReO}_{3} / \mathrm{ReO}_{4}{ }^{-}$Electrode and the Thermodynamics of Rhenium Trioxide. J. Am. Chem. Soc. 1957, 79, 1559-1563. (25) Busey, R. H.; Sprague, E. D.; Bevan, R. B., Enthalpy of hydrolysis of rhenium trichloride. Enthalpy and free energy of formation of rhenium sesquioxide. $J$. Phys. Chem. 1969, 73, 1039-1042.

(26) Beattie, I. R.; Gilson, T. R.; Jones, P. J., Vapor Phase Vibrational Spectra for $\mathrm{Re}_{2} \mathrm{O}_{7}$ and the Infrared Spectrum of Gaseous $\mathrm{HReO}_{4}$. Molecular Shapes of $\mathrm{Mn}_{2} \mathrm{O}_{7}, \mathrm{Tc}_{2} \mathrm{O}_{7}$, and $\mathrm{Re}_{2} \mathrm{O}_{7}$. Inorg. Chem. 1996, 35, 1301-1304.

(27) Meisel, K., Rheniumtrioxyd. III. Mitteilung. Über die Kristallstruktur des Rheniumtrioxyds. Z. Anorg. Allg. Chem. 1932, 207, 121-128.

(28) Hens, Z.; Martins, J. C., A Solution NMR Toolbox for Characterizing the Surface Chemistry of Colloidal Nanocrystals. Chem. Mater. 2013, 25, 12111221.

(29) Greiner Mark, T.; Rocha Tulio, C. R.; Johnson, B.; Klyushin, A.; KnopGericke, A.; Schlögl, R., The Oxidation of Rhenium and Identification of Rhenium Oxides During Catalytic Partial Oxidation of Ethylene: An In-Situ XPS Study. In Z. Phys. Chem., 2014; Vol. 228, p 521.

(30) Hofmann, B. J.; Harms, R. G.; Schwaminger, S. P.; Reich, R. M.; Kühn, F. E., Reactivity of $\mathrm{Re}_{2} \mathrm{O}_{7}$ in aromatic solvents - Cleavage of a $\beta-O-4$ lignin model substrate by Lewis-acidic rhenium oxide nanoparticles. J. Catal. 2019, 373, 190200.

(31) Ishii, M.; Tanaka, T.; Akahane, T.; Tsuda, N., Infrared Transmission Spectra of Metallic $\mathrm{ReO}_{3}$. J. Phys. Soc.Jpn. 1976, 41, 908-912.

(32) Clark, B. D.; DeSantis, C. J.; Wu, G.; Renard, D.; McClain, M. J.; Bursi, L.; Tsai, A.-L.; Nordlander, P.; Halas, N. J., Ligand-Dependent Colloidal Stability Controls the Growth of Aluminum Nanocrystals. J. Am. Chem. Soc. 2019, 141, 1716-1724.

(33) Bohren, C. F.; Huffman, D. R., Absorption and Scattering of Light by Small Particles. Wiley: 2008.

(34) Ehrenreich, H.; Philipp, H. R., Optical Properties of Ag and Cu. Phys. Rev. $1962,128,1622-1629$.

(35) Ehrenreich, H.; Philipp, H. R.; Segall, B., Optical Properties of Aluminum. Phys. Rev. 1963, 132, 1918-1928.

(36) Johnson, P. B.; Christy, R. W., Optical Constants of the Noble Metals. Phys. Rev. B 1972, 6, 4370-4379.

(37) Philipp, H. R.; Ehrenreich, H., Optical Properties of Semiconductors. Phys. Rev. 1963, 129, 1550-1560.

(38) Marton, L., Experiments on Low-Energy Electron Scattering and Energy Losses. Rev. Mod. Phys. 1956, 28, 172-183.

(39) Nozières, P.; Pines, D., Electron Interaction in Solids. Characteristic Energy Loss Spectrum. Phys. Rev. 1959, 113, 1254-1267.

(40) Pankove, J. I., Optical Processes in Semiconductors. Dover: 1975.

(41) Maier, S. A., Electromagnetics of Metals. In Plasmonics: Fundamentals and Applications, Maier, S. A., Ed. Springer US: New York, NY, 2007; pp 5-19.

(42) Maier, S. A., Localized Surface Plasmons. In Plasmonics: Fundamentals and Applications, Maier, S. A., Ed. Springer US: New York, NY, 2007; pp 65-88.

(43) Fröhlich, H., Theory of dielectrics: dielectric constant and dielectric loss. Clarendon Press: 1949.

(44) Gaspari, R.; Della Valle, G.; Ghosh, S.; Kriegel, I.; Scotognella, F.; Cavalli, A.; Manna, L., Quasi-Static Resonances in the Visible Spectrum from AllDielectric Intermediate Band Semiconductor Nanocrystals. Nano Lett. 2017, 17, 7691-7695.

(45) Kreibig, U.; Vollmer, M., Optical properties of metal clusters. Springer: 1995.

(46) Runnerstrom, E. L.; Bergerud, A.; Agrawal, A.; Johns, R. W.; Dahlman, C. J.; Singh, A.; Selbach, S. M.; Milliron, D. J., Defect Engineering in Plasmonic Metal Oxide Nanocrystals. Nano Lett. 2016, 16, 3390-3398.
(47) Chen, H.; Kou, X.; Yang, Z.; Ni, W.; Wang, J., Shape- and Size-Dependent Refractive Index Sensitivity of Gold Nanoparticles. Langmuir 2008, 24, 5233 5237.

(48) Duval Malinsky, M.; Kelly, K. L.; Schatz, G. C.; Van Duyne, R. P. Nanosphere Lithography: Effect of Substrate on the Localized Surface Plasmon Resonance Spectrum of Silver Nanoparticles. J. Phys. Chem. B 2001, 105, $2343-$ 2350.

(49) Mattox, T. M.; Agrawal, A.; Milliron, D. J., Low Temperature Synthesis and Surface Plasmon Resonance of Colloidal Lanthanum Hexaboride $\left(\mathrm{LaB}_{6}\right)$ Nanocrystals. Chem. Mater. 2015, 27, 6620-6624.

(50) Jain, P. K.; Lee, K. S.; El-Sayed, I. H.; El-Sayed, M. A., Calculated Absorption and Scattering Properties of Gold Nanoparticles of Different Size, Shape, and Composition: Applications in Biological Imaging and Biomedicine. J. Phys. Chem. B 2006, 110, 7238-7248.

(51) Liu, X.; Atwater, M.; Wang, J.; Huo, Q., Extinction coefficient of gold nanoparticles with different sizes and different capping ligands. Colloids Surf., B 2007, 58, 3-7.

(52) Paramelle, D.; Sadovoy, A.; Gorelik, S.; Free, P.; Hobley, J.; Fernig, D. G., A rapid method to estimate the concentration of citrate capped silver nanoparticles from UV-visible light spectra. Analyst 2014, 139, 4855-4861.

(53) Link, S.; Wang, Z. L.; El-Sayed, M. A., Alloy Formation of Gold-Silver Nanoparticles and the Dependence of the Plasmon Absorption on Their Composition. J. Phys. Chem. B 1999, 103, 3529-3533.

(54) zum Felde, U.; Haase, M.; Weller, H., Electrochromism of Highly Doped Nanocrystalline $\mathrm{SnO}_{2}$ :Sb. J. Phys. Chem. B 2000, 104, 9388-9395.

(55) Pflughoefft, M.; Weller, H., Spectroelectrochemical Analysis of the Electrochromism of Antimony-Doped Nanoparticulate Tin-Dioxide Electrodes. J. Phys. Chem. B2002, 106, 10530-10534.

(56) Garcia, G.; Buonsanti, R.; Runnerstrom, E. L.; Mendelsberg, R. J.; Llordes, A.; Anders, A.; Richardson, T. J.; Milliron, D. J., Dynamically Modulating the Surface Plasmon Resonance of Doped Semiconductor Nanocrystals. Nano Lett. 2011, 11, 4415-4420.

(57) Dahlman, C. J.; Tan, Y.; Marcus, M. A.; Milliron, D. J. Spectroelectrochemical Signatures of Capacitive Charging and Ion Insertion in Doped Anatase Titania Nanocrystals. J. Am. Chem. Soc. 2015, 137, 9160-9166. (58) Kim, J.; Ong, G. K.; Wang, Y.; LeBlanc, G.; Williams, T. E.; Mattox, T. M.; Helms, B. A.; Milliron, D. J., Nanocomposite Architecture for Rapid, SpectrallySelective Electrochromic Modulation of Solar Transmittance. Nano Lett. 2015 $15,5574-5579$

(59) Dahlman, C. J.; LeBlanc, G.; Bergerud, A.; Staller, C.; Adair, J.; Milliron, D. J., Electrochemically Induced Transformations of Vanadium Dioxide Nanocrystals. Nano Lett. 2016, 16, 6021-6027.

(60) Bashian, N. H.; Zhou, S.; Zuba, M.; Ganose, A. M.; Stiles, J. W.; Ee, A.; Ashby, D. S.; Scanlon, D. O.; Piper, L. F. J.; Dunn, B.; Melot, B. C., Correlated Polyhedral Rotations in the Absence of Polarons during Electrochemical Insertion of Lithium in $\mathrm{ReO}_{3}$. ACS Energy Lett. 2018, 3, 2513-2519.

(61) Cava, R. J.; Santoro, A.; Murphy, D. W.; Zahurak, S.; Roth, R. S., The structures of lithium-inserted metal oxides: $\mathrm{LiReO}_{3}$ and $\mathrm{Li}_{2} \mathrm{ReO}_{3}$. J. Solid State Chem. 1982, 42, 251-262.

(62) Wang, J.; Polleux, J.; Lim, J.; Dunn, B., Pseudocapacitive Contributions to Electrochemical Energy Storage in $\mathrm{TiO}_{2}$ (Anatase) Nanoparticles. J. Phys. Chem. C 2007, 111, 14925-14931.

(63) Ung, T.; Giersig, M.; Dunstan, D.; Mulvaney, P., Spectroelectrochemistry of Colloidal Silver. Langmuir 1997, 13, 1773-1782.

(64) Novo, C.; Funston, A. M.; Gooding, A. K.; Mulvaney, P., Electrochemical Charging of Single Gold Nanorods. J. Am. Chem. Soc. 2009, 131, 14664-14666. (65) Granqvist, C. G., Chapter 22 - Miscellaneous Oxide Films. In Handbook of Inorganic Electrochromic Materials, Granqvist, C. G., Ed. Elsevier Science B.V.: Amsterdam, 1995; pp 401-412. 
TOC Graphics

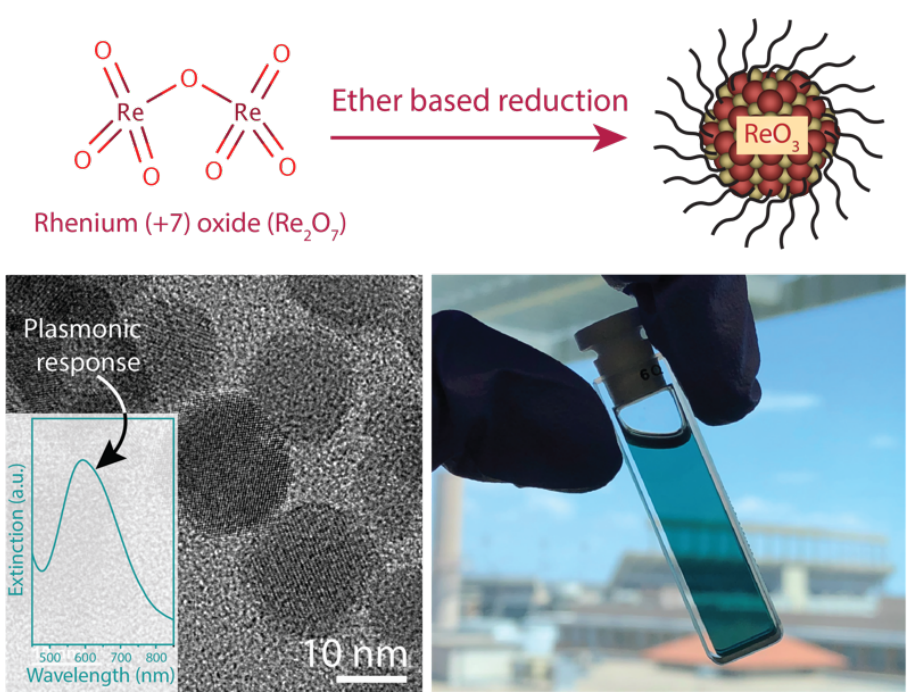




\section{Supporting Information}

\section{Colloidal $\mathrm{ReO}_{3}$ Nanocrystals: Extra $\mathrm{Re}$ d-electron instigating a plasmonic response}

Sandeep Ghosh ${ }^{\dagger}$, Hsin-Che $\mathrm{Lu}^{+}$, Shin Hum $\mathrm{Cho}^{+}$, Thejaswi Maruvada ${ }^{\dagger}$, Murphie C. Price ${ }^{\dagger}$, and Delia J. Milliron ${ }^{+, *}$

' McKetta Department of Chemical Engineering, The University of Texas at Austin, Austin, Texas 78712-1589, United States

Corresponding Author

*E-mail: milliron@che.utexas.edu 


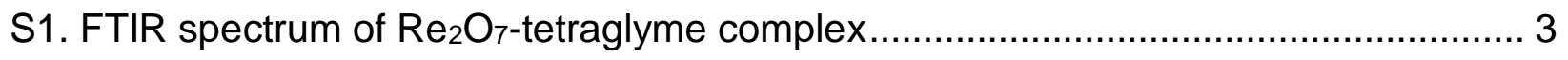

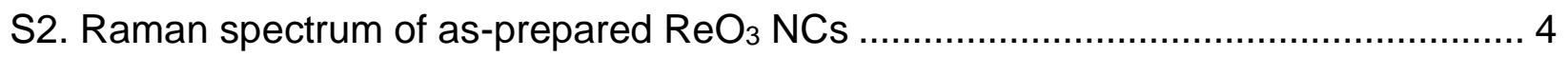

S3. Brown amorphous product (synthesis with alcohols, amines, or carboxylic acids) ... 5

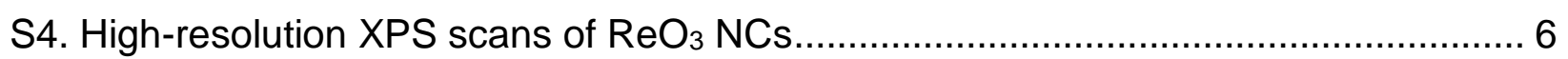

$\mathrm{S} 5$. Photographs of solutions of $\mathrm{Re}_{2} \mathrm{O}_{7}$ in tetraglyme and dioctyl ether ........................ 7

S6. Proposed reaction intermediate resulting in formation of surface hydroxyls ............. 8

S7. NMR and FTIR spectra of the synthesis mixture ................................................. 9

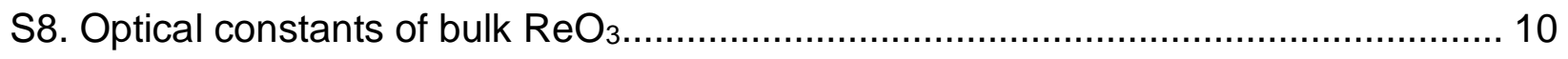

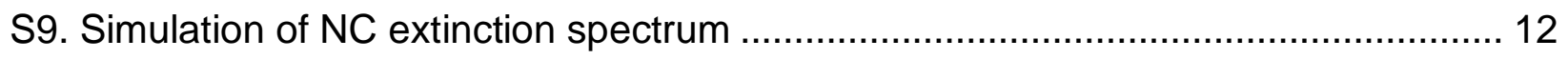

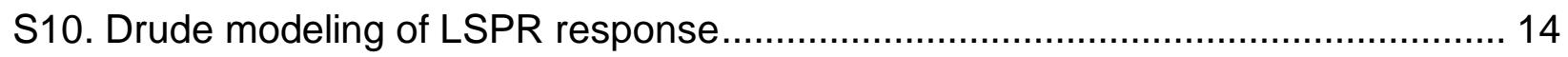

S11. Optical extinction spectra showing effects of aggregation on LSPR ...................... 15

S12. Reversibility of cyclic voltammetry (CV) scans for Li- and TBA-ions .................... 16

S13. Perovskite crystal structure of $\mathrm{ReO}_{3}$ showing missing A-site cation........................ 17

S14. High-resolution XPS scan of $\mathrm{ReO}_{3} \mathrm{NCs}$ on Li-foil ........................................... 18

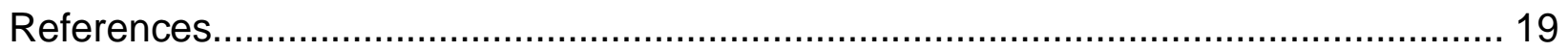




\section{S1. FTIR spectrum of $\mathrm{Re}_{2} \mathrm{O}_{7}$-tetraglyme complex}

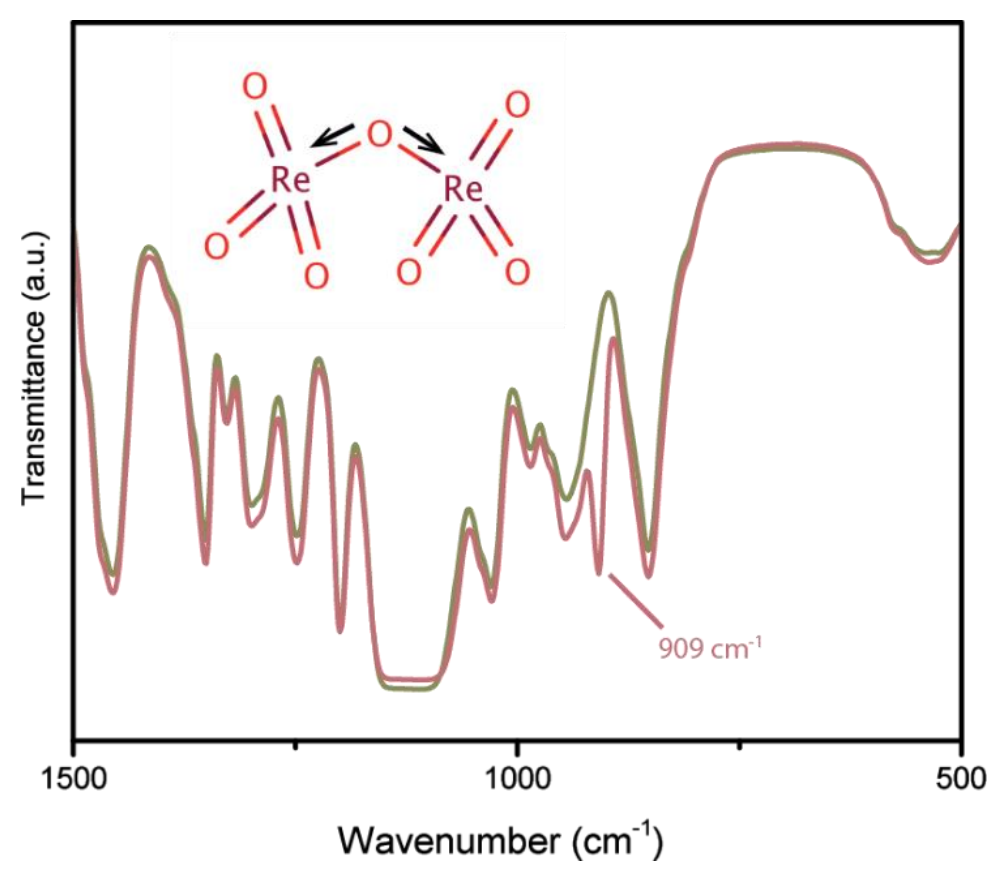

Figure S1. FTIR spectrum of $\operatorname{Re}_{2} \mathrm{O}_{7}$ in tetraglyme (red curve), in comparison to the tetraglyme spectrum (green curve). The Re-O-Re stretching mode (shown in inset) is marked at $909 \mathrm{~cm}^{-1}$. 


\section{S2. Raman spectrum of as-prepared $\mathrm{ReO}_{3} \mathrm{NCs}$}

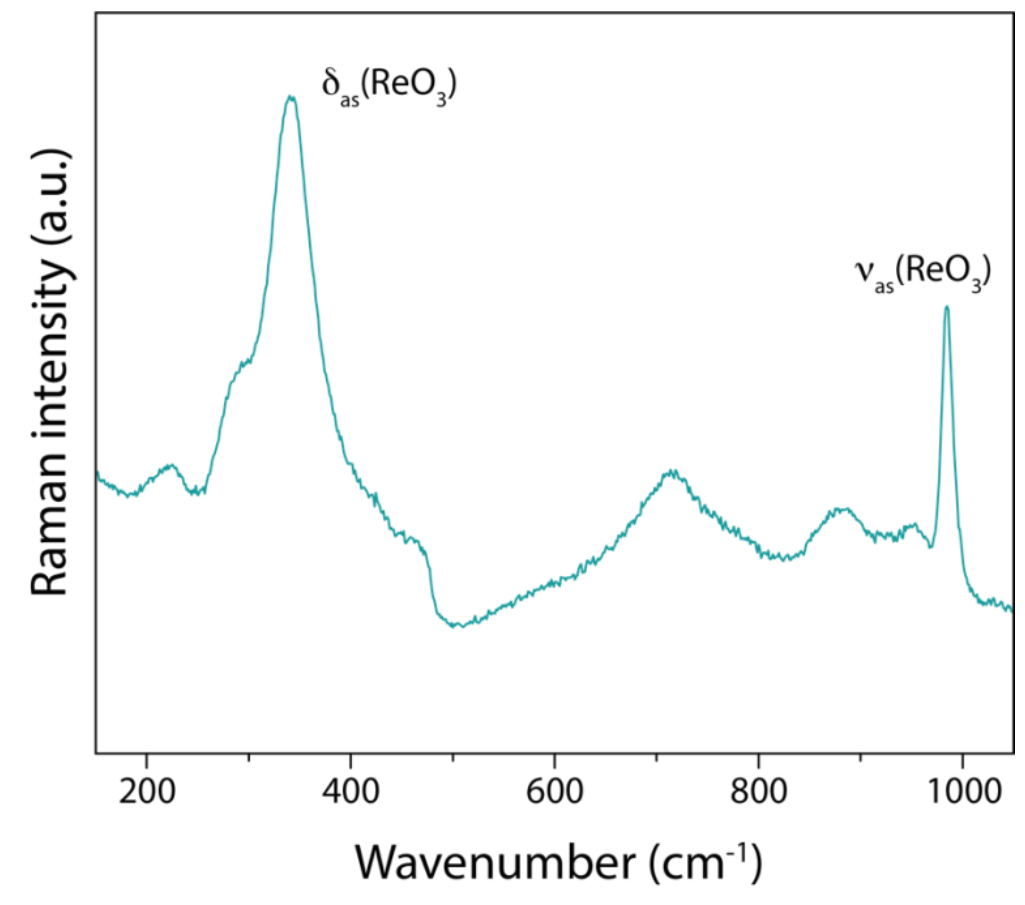

Figure S2. Raman spectrum of as-prepared $\mathrm{ReO}_{3} \mathrm{NCs}$, showing the characteristics asymmetric stretching $\left(v_{\text {as }}\right)$ and bending $\left(\delta_{\text {as }}\right)$ modes of the crystal phase. ${ }^{1}$ The spectrum was collected on a dropcast film of $\mathrm{ReO}_{3} \mathrm{NCs}$ using Horiba LabRAM Aramis instrument equipped with a confocal aperture, at $632 \mathrm{~nm}$ excitation wavelength and acquisition time of $180 \mathrm{~s}$. 
S3. Brown amorphous product (synthesis with alcohols, amines, or carboxylic acids)

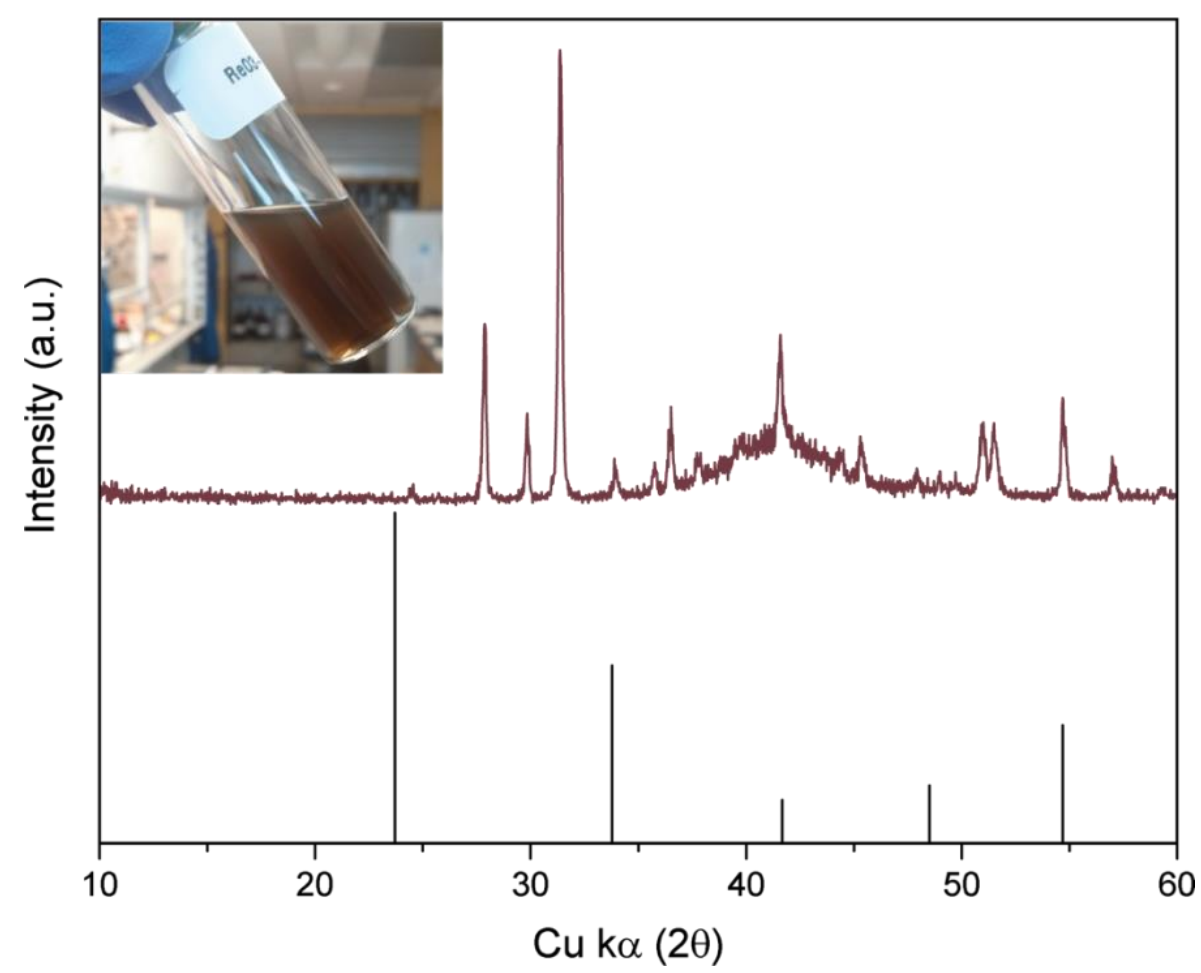

Figure S3. XRD pattern of the brown amorphous product when the NC synthesis was performed in the presence of oleyl alcohol, in reference to database powder XRD line pattern of $\mathrm{ReO}_{3}$ (AMCSD \#0016967). Photograph of the product in the inset. This product is representative of those formed with long chain alcohols, amines or alkylcarboxylic acids. 


\section{S4. High-resolution XPS scans of $\mathrm{ReO}_{3} \mathrm{NCs}$}
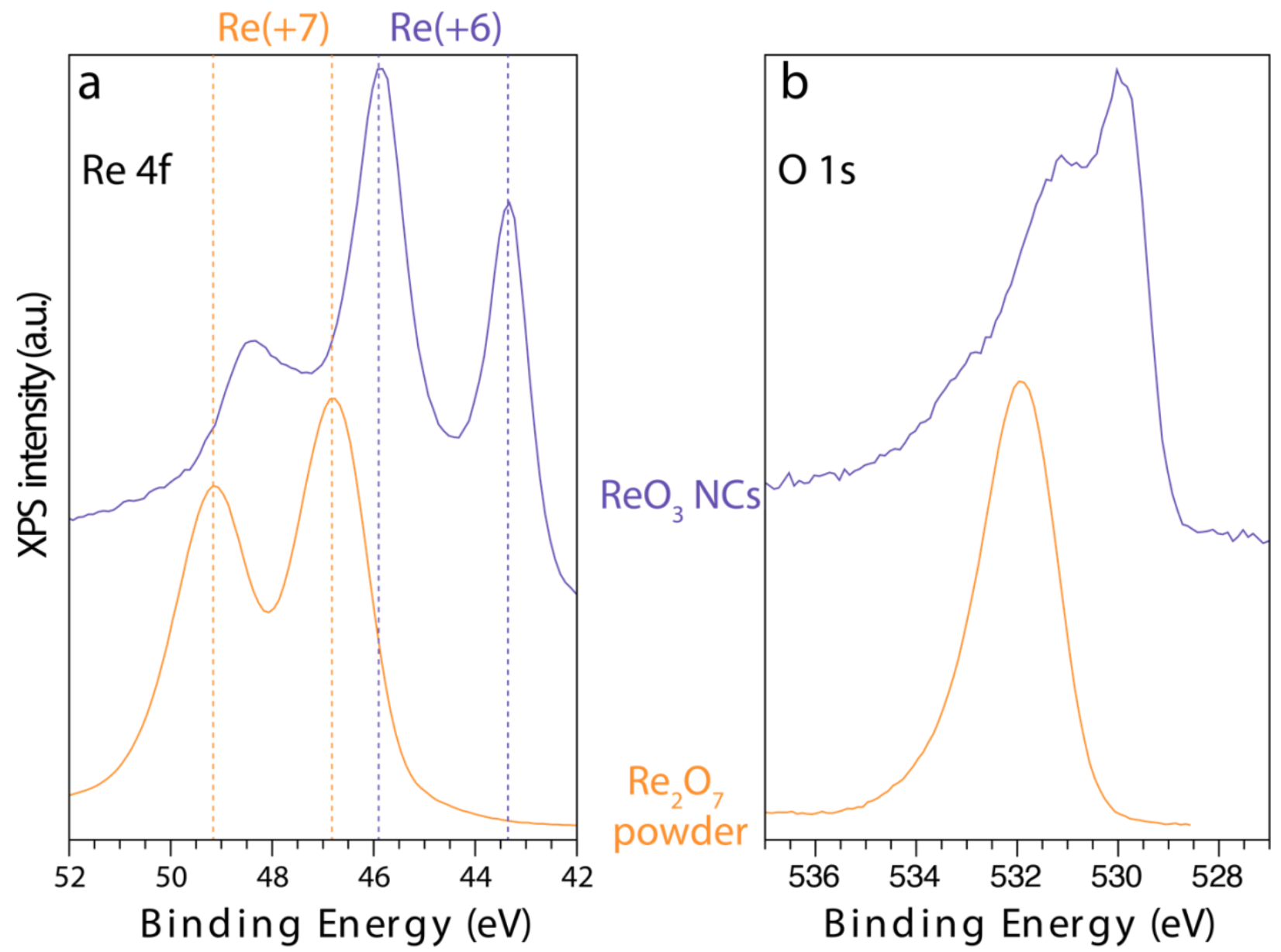

Figure S4. Re 4f (panel a) and O 1s (panel b) high-resolution XPS scans of the as-synthesized $\mathrm{ReO}_{3} \mathrm{NCs}$ in comparison to those of commercially procured $\mathrm{Re}_{2} \mathrm{O}_{7}$ powder. The $\mathrm{Re} 4 \mathrm{f}$ signals due to +7 and +6 oxidation states are marked in panel a. The triplet observed in $\mathrm{ReO}_{3} \mathrm{NCs}$ is due to convoluted signals from $\operatorname{Re}(+6)$ doublets and the surface hydroxyls leading to an ill-defined oxidation state for surface Re atoms (a combination of +7 and +6 oxidation states). 


\section{S5. Photographs of solutions of $\mathrm{Re}_{2} \mathrm{O}_{7}$ in tetraglyme and dioctyl ether}

a. $\mathrm{Re}_{2} \mathrm{O}_{7}$ in tetraglyme

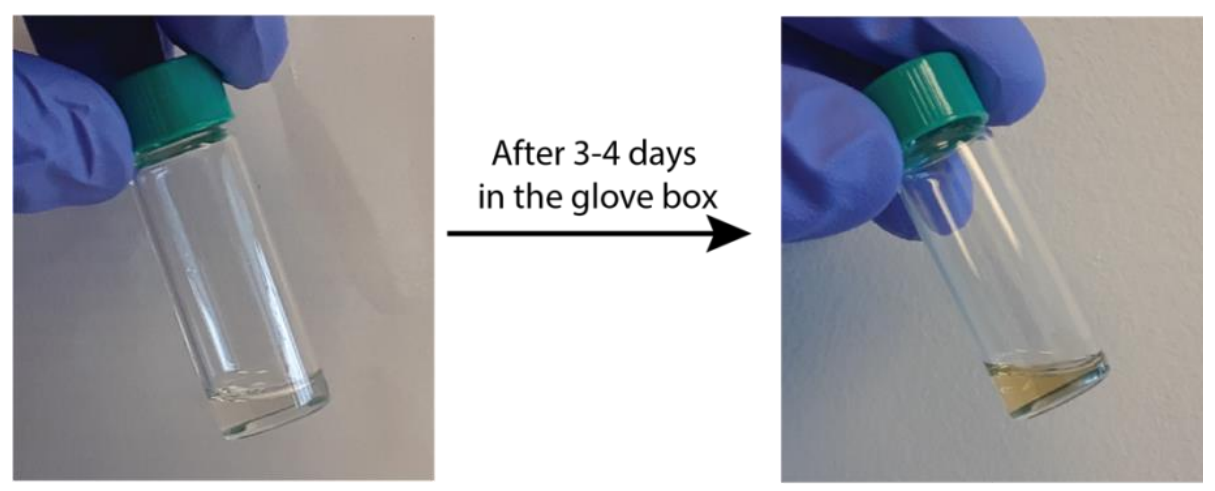

b. $\mathrm{Re}_{2} \mathrm{O}_{7}$ in dioctyl ether

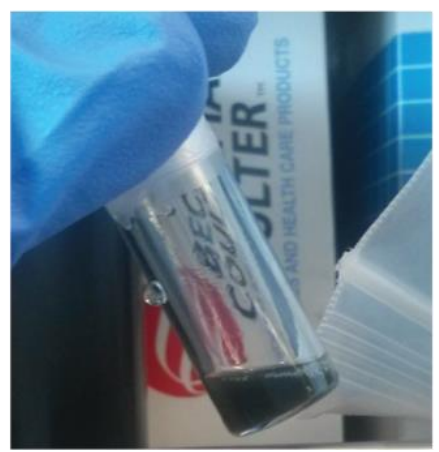

Figure S5. Photographs showing the effects of tetraglyme and dioctyl ether on the $\operatorname{Re}_{2} \mathrm{O}_{7}$ precursor. The solution of $\mathrm{Re}_{2} \mathrm{O}_{7}$ in tetraglyme slowly turns brown, which indicates that this solution should be freshly prepared before injection. Dioctyl ether, on the other hand, turns $\operatorname{Re}_{2} \mathrm{O}_{7}$ green almost instantaneously, rendering it an ideal reductant in a hot injection synthesis. 
S6. Proposed reaction intermediate resulting in formation of surface hydroxyls

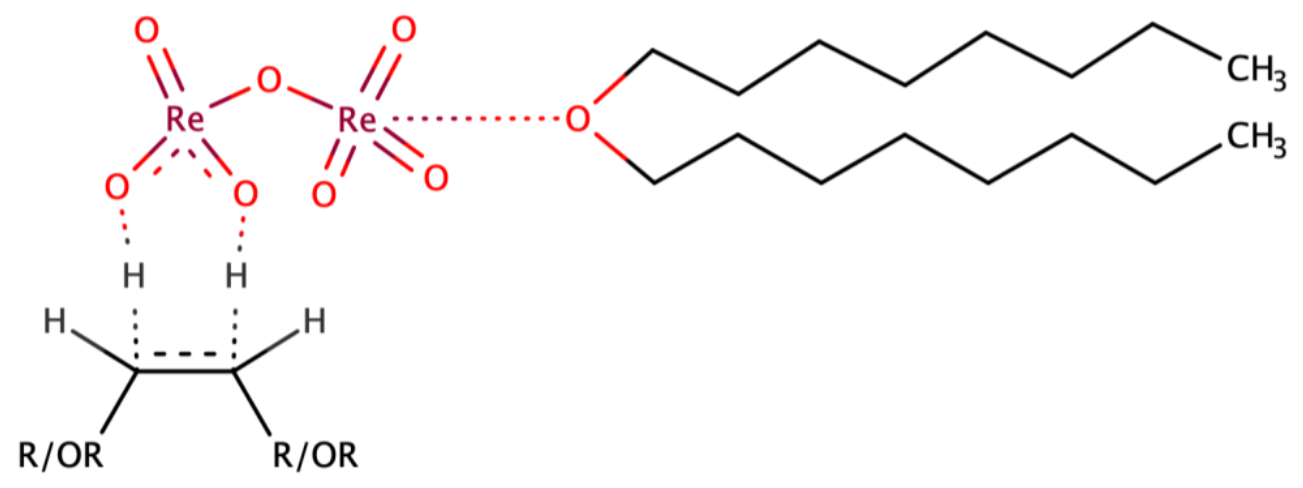

Figure S6. Sketch of a plausible reaction intermediate that can explain the formation of hydroxyl moieties on the $\mathrm{ReO}_{3} \mathrm{NC}$ surfaces and the observation of alkene protons in NMR spectrum (Figure 2 , main text). 


\section{S7. NMR and FTIR spectra of the synthesis mixture}
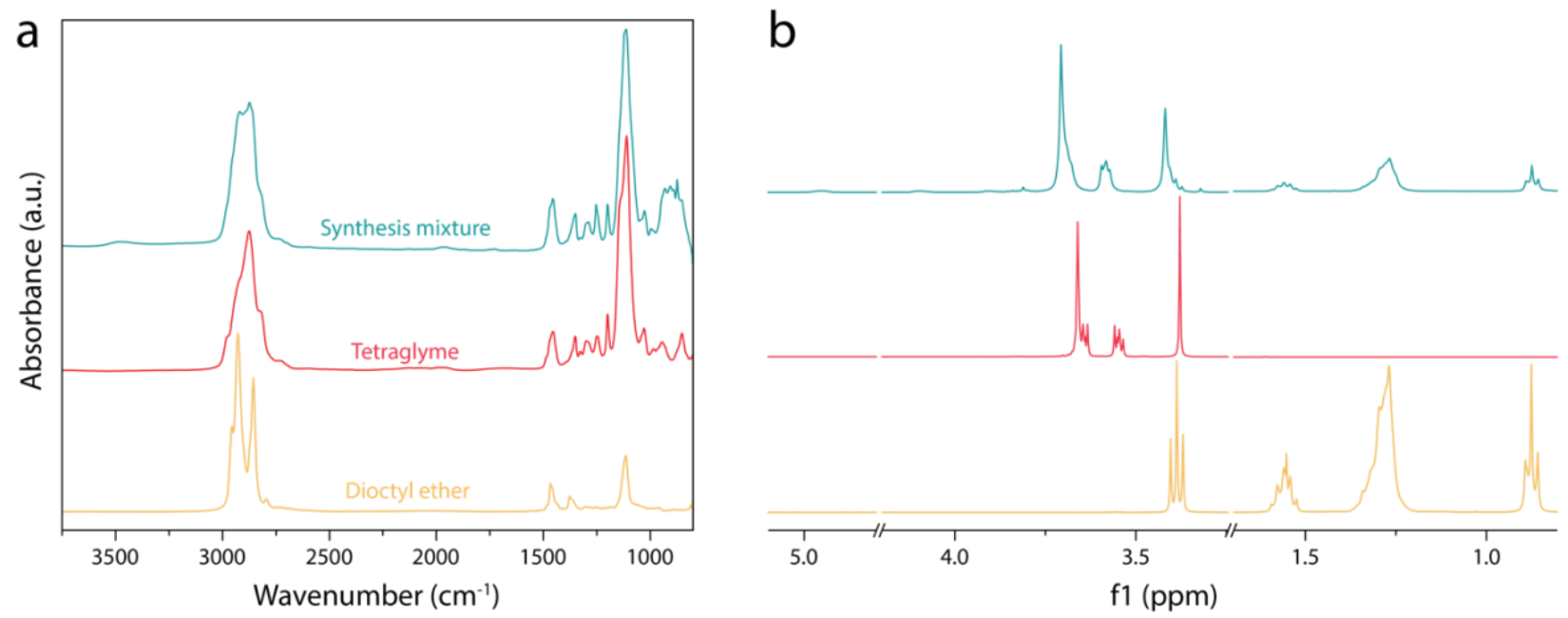

Figure S7. Wider range FTIR (panel a) and NMR (panel b) spectra of the synthesis mixture (blue curves) in comparison to that for TGY (red curve) and DOE (yellow curve). 


\section{S8. Optical constants of bulk $\mathrm{ReO}_{3}$}

The real and imaginary parts of the dielectric function ( $\varepsilon^{\prime}$ and $\varepsilon^{\prime \prime}$, respectively) for $\mathrm{ReO}_{3}$ were extracted from the data reported by Feinleib et al. obtained from absolute reflectance measurements on a single-crystal of $\mathrm{ReO}_{3} .{ }^{2}$ Data extraction was performed using the freely available web-based program, WEBPLOTDIGITIZER (https://automeris.io/WebPlotDigitizer, author: Ankit Rohatgi, Ver: 4.1, Jan, 2018). The real and imaginary parts of the refractive index ( $\eta$ and $\kappa$, respectively) for $\mathrm{ReO}_{3}$ were then calculated using the following equations:

$$
\begin{gathered}
\eta=\sqrt{\frac{\sqrt{{\varepsilon^{\prime 2}+\varepsilon^{\prime 2}}^{2}}+\varepsilon^{\prime}}{2}} \\
\kappa=\sqrt{\frac{\sqrt{{\varepsilon^{\prime 2}+\varepsilon^{\prime 2}}^{2}-\varepsilon^{\prime}}}{2}}
\end{gathered}
$$

The plots for $\varepsilon^{\prime}$ and $\varepsilon^{\prime \prime}$ are shown in Figure S8a and S9a, and that for $\eta$ and $\kappa$, in Figures S9b below. The optical absorption coefficient $(\alpha)$ was then calculated using the following formula:

$$
\alpha=\frac{4 \pi \kappa}{\lambda}
$$

The absorption coefficient is plotted in Figure S9c.

The loss function is defined as $-\operatorname{Im}\left(\varepsilon^{-1}\right)$ or in other form as $\varepsilon^{\prime \prime} /\left(\varepsilon^{\prime 2}+\varepsilon^{\prime 2}\right)$, and is plotted in Figures S8c and S9c - peaks in this curve are associated with plasma oscillations.
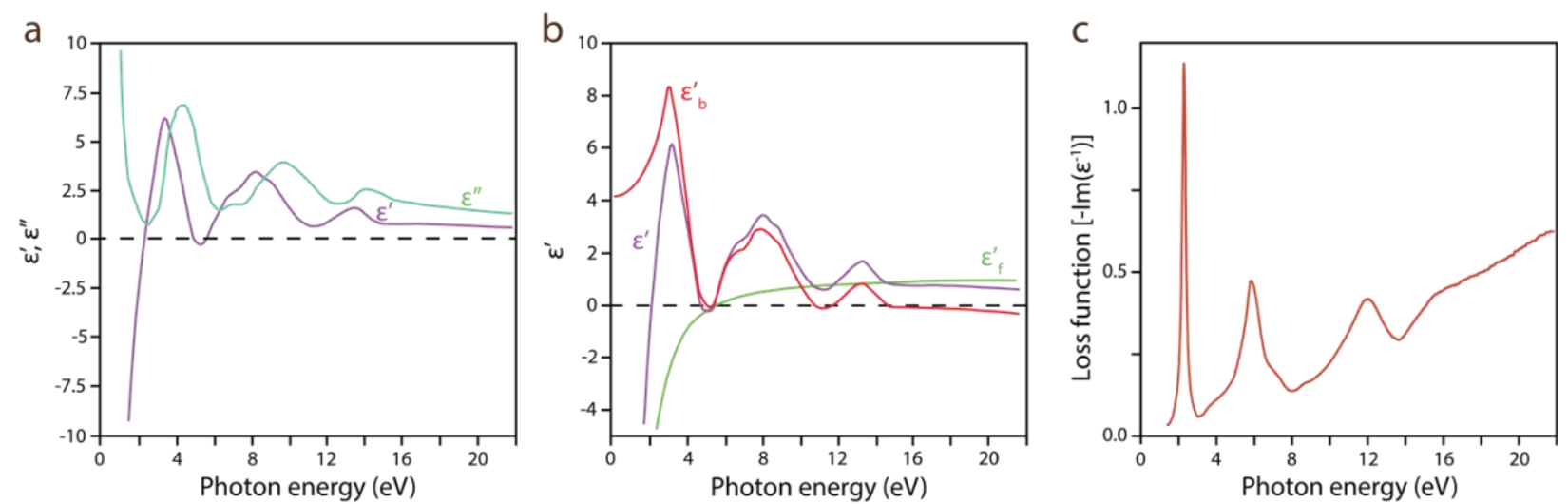

Figure S8. Optical characteristics of an $\mathrm{ReO}_{3}$ single-crystal over the entire energy range. ${ }^{2}$ (a)real (purple) and imaginary (green) parts of the dielectric function, (b) real part of the dielectric function (purple curve) deconvoluted into bound (red curve) and free electron (green curve) contributions, and (c) loss function (red curve). 


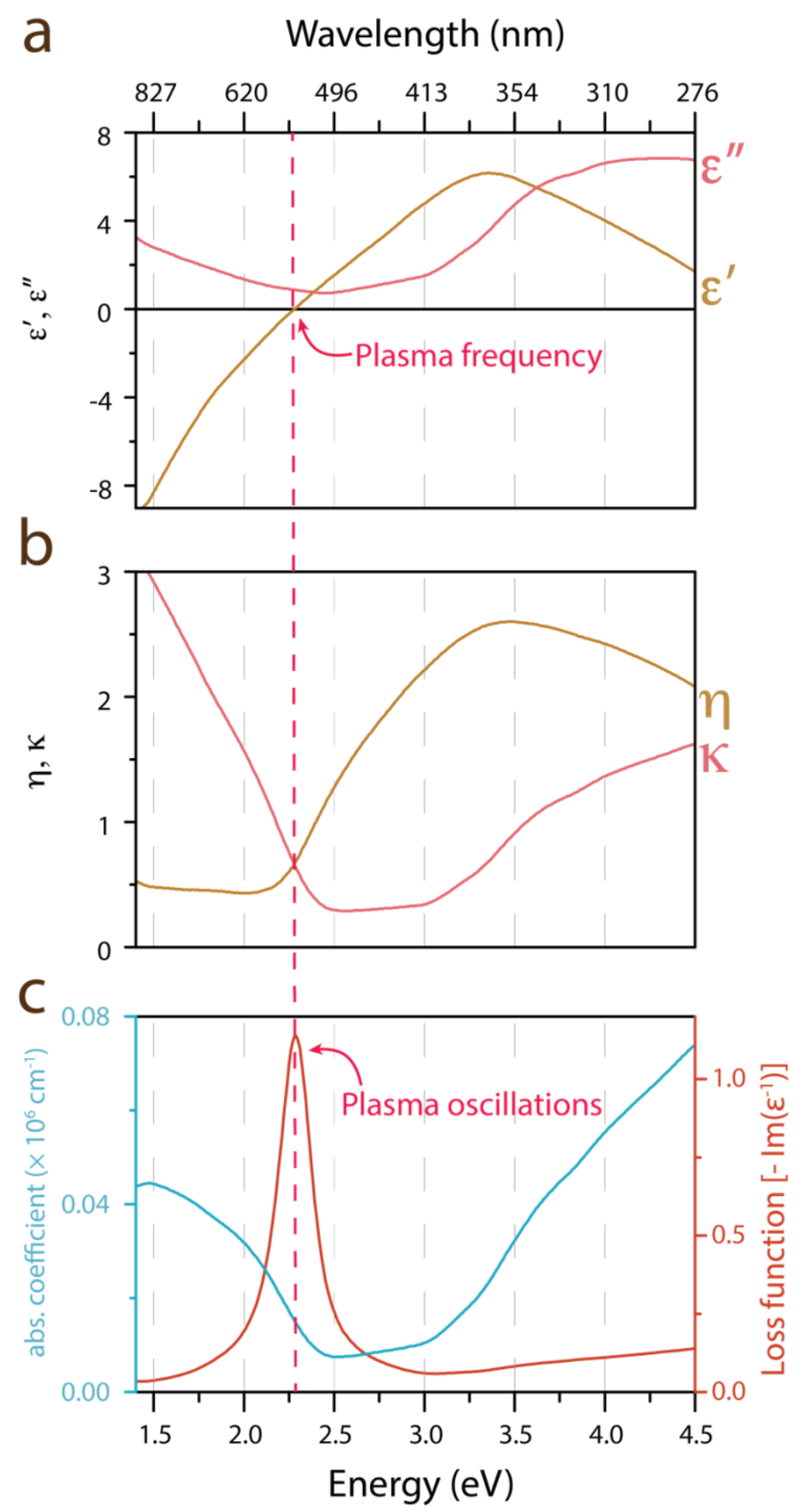

Figure S9. Optical characteristics of an $\mathrm{ReO}_{3}$ single-crystal: ${ }^{2}$ (a) real and imaginary parts of the dielectric function, (b) real and imaginary parts of the refractive index calculated from the respective dielectric functions, and (c) bulk absorption coefficient (blue curve) calculated from the imaginary part of the refractive index, along with the loss function (red curve). 


\section{S9. Simulation of NC extinction spectrum}

The COMSOL multiphysics program (https://www.comsol.com) was used to calculate the extinction spectrum for $\mathrm{ReO}_{3} \mathrm{NCs}$ from the bulk values of the real and imaginary parts of the dielectric function measured from the reflectivity of a single crystal of $\mathrm{ReO}_{3} .{ }^{2}$ The computation uses the electrostatic approximation for the interaction of a conductive sphere, a few nanometers across, with an external electric field. ${ }^{3-4}$ Since, localized surface plasmon resonance (LSPR) is a non-propagating excitation of the free charge carriers (electrons/holes) in the metallic nanostructures coupled to the electromagnetic (EM) field, the phase of an oscillating field over a sphere of diameter (d) much smaller than wavelength $(\lambda)$ of light is practically constant. This is called the quasi-static approximation (or limit) and is valid at $d<<$, where the spatial variation in the field can be ignored and the problem can be simplified to that of a nanosphere in an "electrostatic" field.

The geometry for this analytical treatment includes a homogeneous isotropic sphere of radius (r) located at the origin in a uniform electric field, with a non-absorbing isotropic surrounding medium (with dielectric constant $\varepsilon_{m}$ ) and the field lines being parallel to the $\mathrm{z}$-direction at sufficient distance from the sphere. A lowest order approximation of the full scattering problem can then describe the optical characteristics of nanoparticles of sizes below $100 \mathrm{~nm}$ adequately. The Mie solutions to this scattering problem yields an expression for the dipole polarizability $(\alpha)$ of the nanosphere:

$$
\alpha=4 \pi r^{3} \frac{\varepsilon-\varepsilon_{m}}{\varepsilon+2 \varepsilon_{m}}
$$

Here, $\varepsilon$ is the dielectric function of the $\mathrm{NC}$ core material $\left(\mathrm{ReO}_{3}\right.$ in the present case). As is apparent from eq 10.1 above, a resonant enhancement occurs in $\alpha$ when the denominator vanishes- this is called the Fröhlich condition and the associated optical mode for a metallic NC like that of $\mathrm{ReO}_{3}$ is called the LSPR. It is important to note that this resonance red-shifts as $\varepsilon_{m}$ increases - a characteristic feature of an LSPR used for optical sensing. ${ }^{5}$ The extinction cross-section $\left(\sigma_{\text {ext }}\right)$ is then expressed as the sum of absorption $\left(\sigma_{a b s}\right)$ and scattering $\left(\sigma_{e x t}\right)$ cross-sections i.e. $\sigma_{e x t}=\sigma_{a b s}$ $+\sigma_{s c a}$. The following (eqs 10.2 and 10.3) are the expressions for $\sigma_{a b s}$ and $\sigma_{e x t}$ in terms of $\alpha$, where $\kappa$ is the wave-vector of the incident light (EM field).:

$$
\begin{gathered}
\sigma_{a b s}=\kappa \operatorname{Im}[\alpha]=4 \pi \kappa r^{3} \operatorname{Im}\left[\frac{\varepsilon-\varepsilon_{m}}{\varepsilon+2 \varepsilon_{m}}\right] \\
\sigma_{s c a}=\frac{\kappa^{4}}{6 \pi}|\alpha|^{2}=\frac{8 \pi}{3} \kappa^{4} r^{6}\left|\frac{\varepsilon-\varepsilon_{m}}{\varepsilon+2 \varepsilon_{m}}\right|^{2}
\end{gathered}
$$




\section{Building the model in COMSOL:}

$\mathrm{ReO}_{3}$ sphere of $2.5 \mathrm{~nm}$ in radius was designed using the Wave Optics Module in COMSOL. A surrounding medium was represented by a larger concentric sphere, signifying the solvent chloroform $\left(\varepsilon_{m}=2.08\right)$ provided by built-in Optical Materials Database dielectric constant. ${ }^{6}$ The entire system was then immersed in a perfect index matching layer (PML) which prevents unwanted reflections from outside boundary. Further semi-hemispheric symmetry with perpendicular perfect electrical conductor (PEC) and perfect magnetic conductor (PMC) boundary layer was employed at $\mathrm{NC}$ core and surrounding medium to optimize computation time. The maximum and minimum mesh sizes were set at 0.5 and $0.05 \mathrm{~nm}$, respectively. This enabled extrafine physics-controlled meshing and yielded 438,350 degrees of freedom and corresponded to 5.7 GB RAM for the biconjugate gradient stabilized method (BiCGStab) solver. The finite element method solutions to the Maxwell equations were then obtained in the full field mode for the scattered field formulation with the background electric field propagating along the $\mathrm{x}$-axis and polarized along the z-axis.
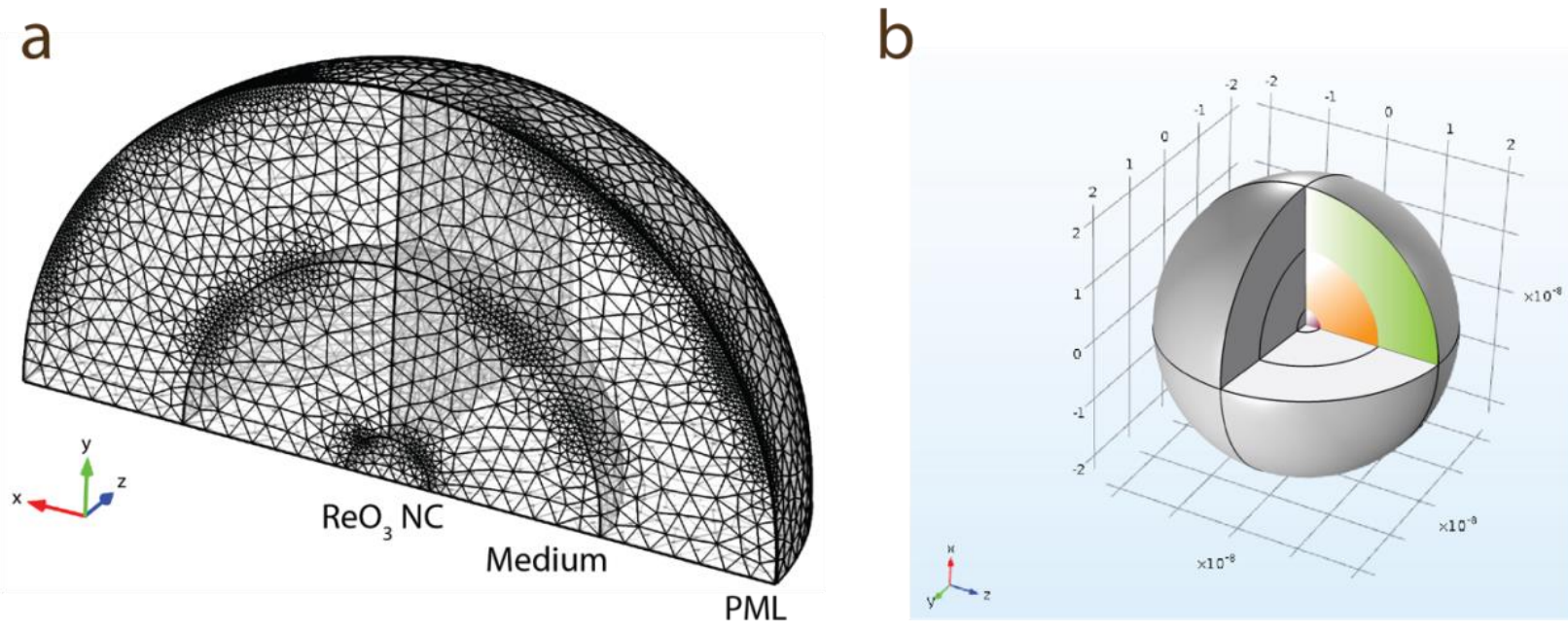

Figure S10. Model for COMSOL computation. (a) Geometry and meshing of the spherical $\mathrm{ReO}_{3}$ $\mathrm{NC}$, medium, and perfect matching layer (PML) boundary conditions for solving the Maxwell equations. b) COMSOL component model schematic for $\mathrm{ReO}_{3} \mathrm{NC}$ (purple core), medium (orange middle layer), and PML boundary (green outer layer). Directional axis is included in inset, with electric field propagation along $\mathrm{x}$-axis and $\mathrm{E}$ field polarization along $\mathrm{z}$-axis. 


\section{S10. Drude modeling of LSPR response}

Since the Drude model is the application of kinetic theory of gases on the gas of "free electrons", it is appropriate to treat the optical response originating from metallic NCs like those of $\mathrm{ReO}_{3}$ under its assumptions and results. ${ }^{7}$ However, the interband transitions in $\mathrm{ReO}_{3}$ can best be treated by the Lorentz oscillator model of band-to-band transition. As mentioned in the main text, the dielectric function of $\mathrm{ReO}_{3}$ has contributions from both free electrons and interband transitions in the system and they have considerable overlap which perturbs the optical response, as shown by Feinleib et al. ${ }^{2}$ Ideally, a complete treatment of the optical extinction should be performed with a combined Drude-Lorentz dispersion model. ${ }^{3}$ However, in order to extract meaningful quantitative information out of the LSPR response of the $\mathrm{ReO}_{3} \mathrm{NCs}$, we have fitted that part of the spectrum (500-850 nm) using the Drude methodology. The dielectric function for a NC of a conductive material like $\mathrm{ReO}_{3}$, as per the Drude contribution $\left(\varepsilon_{D}(\omega)\right)$, is given by the following eq 11.1:

$$
\varepsilon_{D}(\omega)=\varepsilon_{\infty}-\frac{\omega_{p}^{2}}{\omega^{2}+i \omega \Gamma}
$$

Here, $\varepsilon_{\infty}$ is the high-frequency dielectric (which is fixed at 1, as per Figure S8 above), $\omega_{P}$ is the bulk plasma frequency given by eq 11.2 and $\Gamma$ is the electronic damping constant.

$$
\omega_{p}^{2}=\frac{n e^{2}}{\varepsilon_{0} m^{*}}
$$

Here, $n$ is the free charge carrier (electron) density, $e$ being the electronic charge, $\varepsilon_{0}$ the permittivity of vacuum and $m *$ the electron effective mass.

Our Matlab codes used eqs 11.1 and 11.2 above to perform a least-squares fit to the collected spectra and extract the plasma frequency $\left(\omega_{P}\right)$, damping constant $(\Gamma)$ and finally the electron density.

\begin{tabular}{cccccc}
\hline Solvent & $\omega_{P}\left(\mathrm{~cm}^{-1}\right)$ & $\omega_{P}(\mathrm{eV})$ & $\Gamma\left(\mathrm{cm}^{-1}\right)$ & $\Gamma(\mathrm{eV})$ & $\mathrm{n}\left(\times 10^{22} \mathrm{~cm}^{-3}\right)$ \\
\hline Hexane & 37575.7 & 4.66 & 5592.85 & 0.69 & 1.58 \\
Chloroform & 37893.8 & 4.69 & 5167.82 & 0.64 & 1.61 \\
Toluene & 38310.3 & 4.75 & 5245.65 & 0.65 & 1.64 \\
Carbon disulfide & 39574.7 & 4.9 & 5129.62 & 0.64 & 1.75 \\
\hline
\end{tabular}




\section{S11. Optical extinction spectra showing effects of aggregation on LSPR}
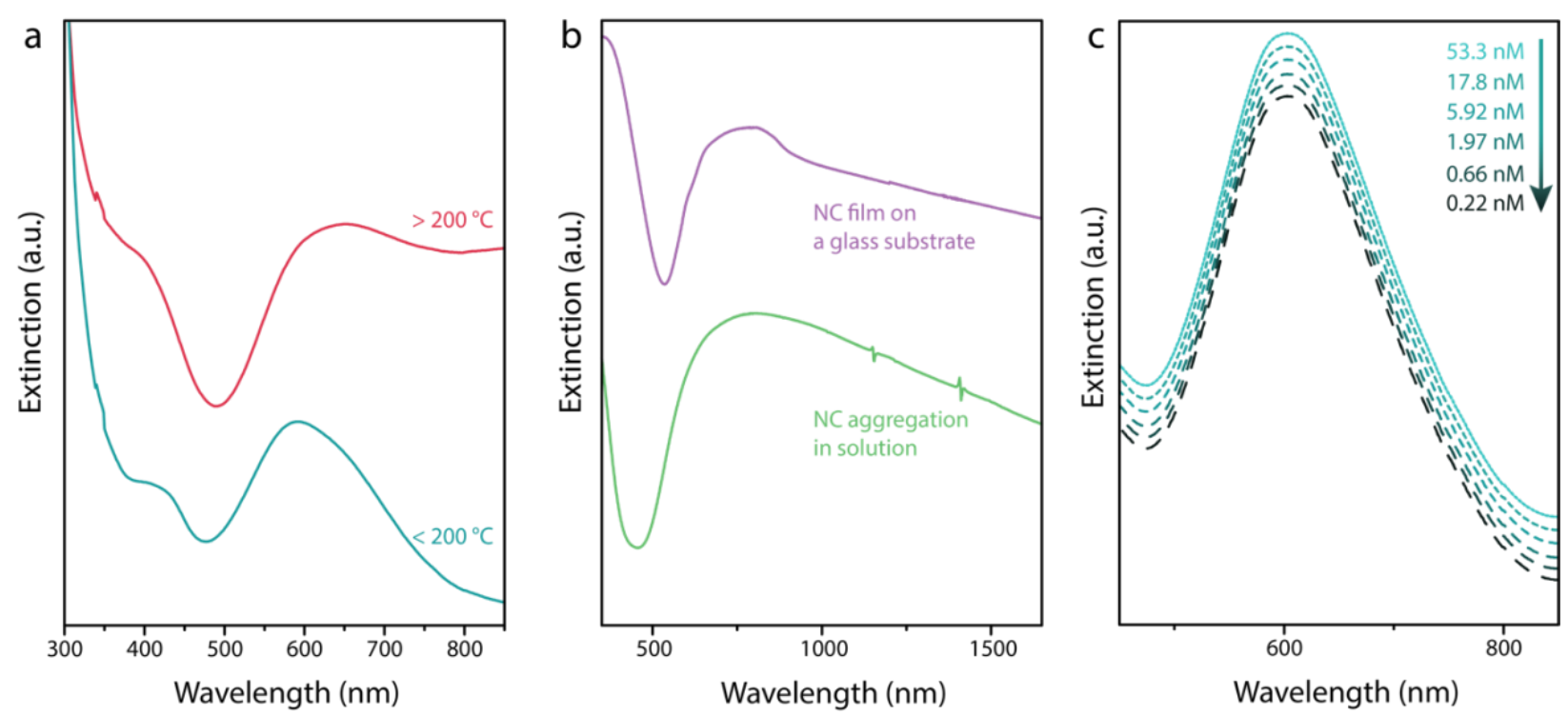

Figure S11. UV-Vis-NIR extinction spectra of $\mathrm{ReO}_{3} \mathrm{NCs}$ : (a) synthesized at less than (blue curve) and more than (red curve) $200{ }^{\circ} \mathrm{C}$, and (b) after multiple washings exhibiting red-shifted curve (green curve) and drop-cast on a glass substrate (purple curve) showing significant red-shift from the original $590 \mathrm{~nm}$ LSPR position of the individually suspended NCs. The considerable shift is attributed to increased LSPR coupling upon aggregation and scattering contributions to extinction. (c) Normalized extinction curves for the dilution series used for the calculation of molar attenuation coefficient in Figure 3. 


\section{S12. Reversibility of cyclic voltammetry (CV) scans for Li- and TBA- ions}
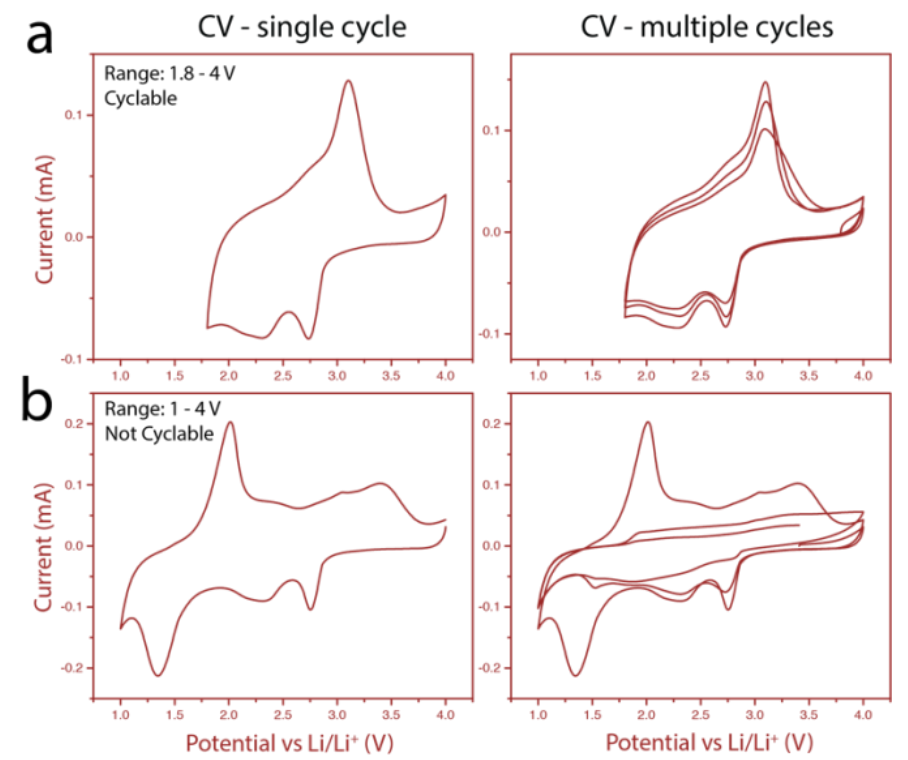

Figure S12. CV scans for Li-ions for different potential ranges with single scans shown on the left panel and multiple scans (depicting reversibility or its lack thereof) on the right panel for each range. (a) $1.8-4 \mathrm{~V}$ (reversible), and (b) $1-4 \mathrm{~V}$ (irreversible). Note that the CV scans in the right panel in (b) become irreversible after the first cycle signaling structure breakdown of the $\mathrm{ReO}_{3}$ lattice.
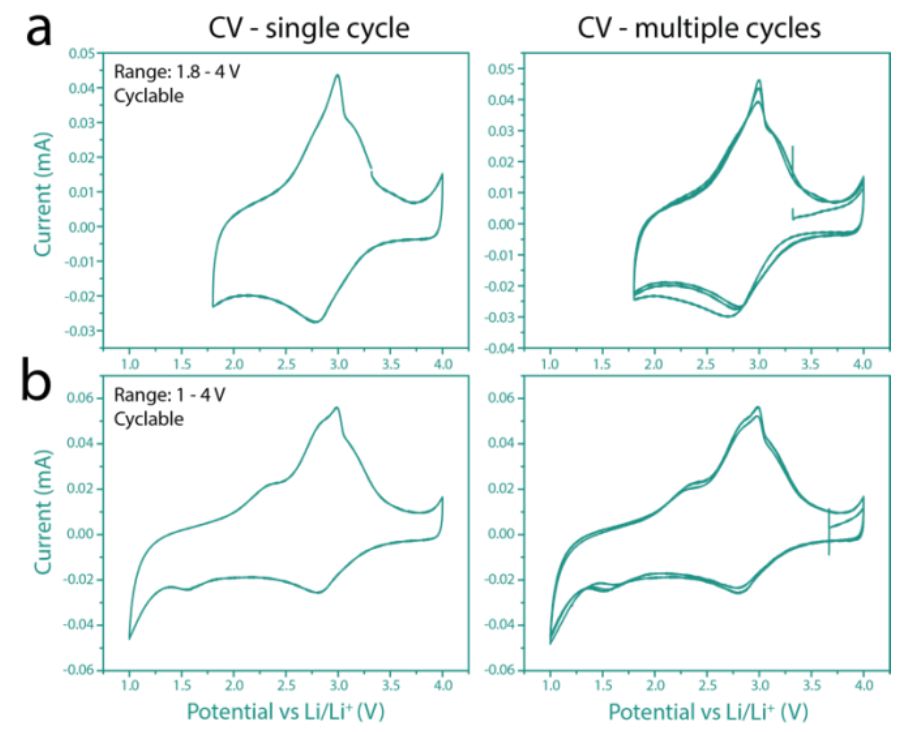

Figure S13. CV scans for TBA-ions for different potential ranges with single scans shown on the left panel and multiple scans on the right panel for each range. (a) $1.8-4 \mathrm{~V}$, (b) $1-4 \mathrm{~V}$. Unlike Li-cycling, TBA-cycling is reversible in the full potential range used for cycling. 


\section{S13. Perovskite crystal structure of $\mathrm{ReO}_{3}$ showing missing A-site cation}

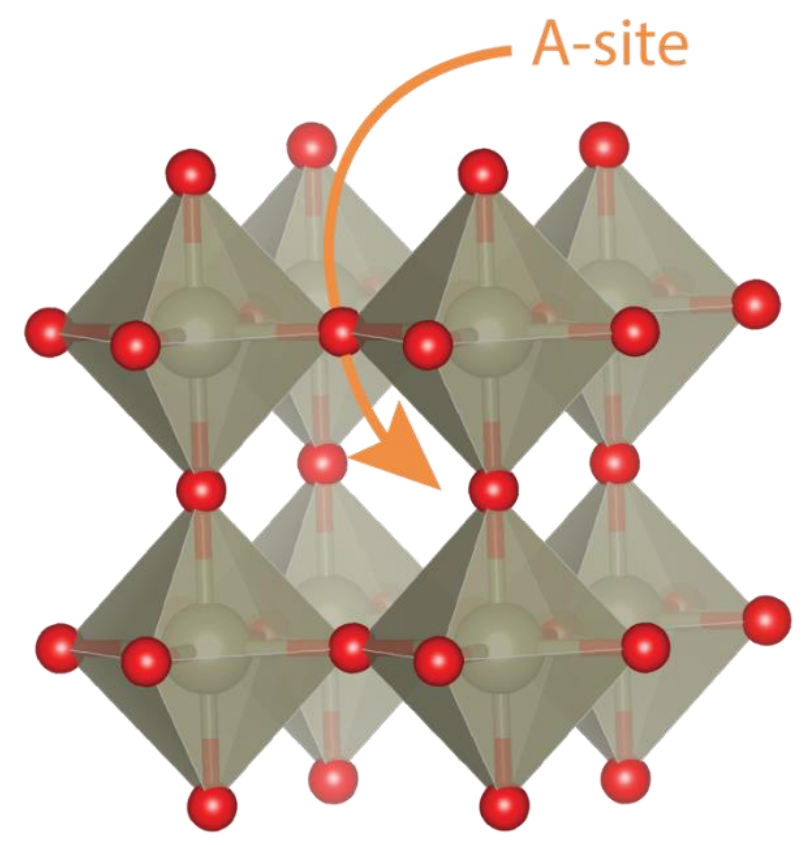

Figure S14. Crystal structure showing the open framework of $\mathrm{ReO}_{3}$ due to the missing large Asite cation from the perovskite $\mathrm{ABO}_{3}$ structure. (Oxygen atoms = red; Rhenium atoms = grey). All the crystal drawings in this manuscript were produced using the VESTA software. ${ }^{8}$ 


\section{S14. High-resolution XPS scan of $\mathrm{ReO}_{3} \mathrm{NCs}$ on Li-foil}

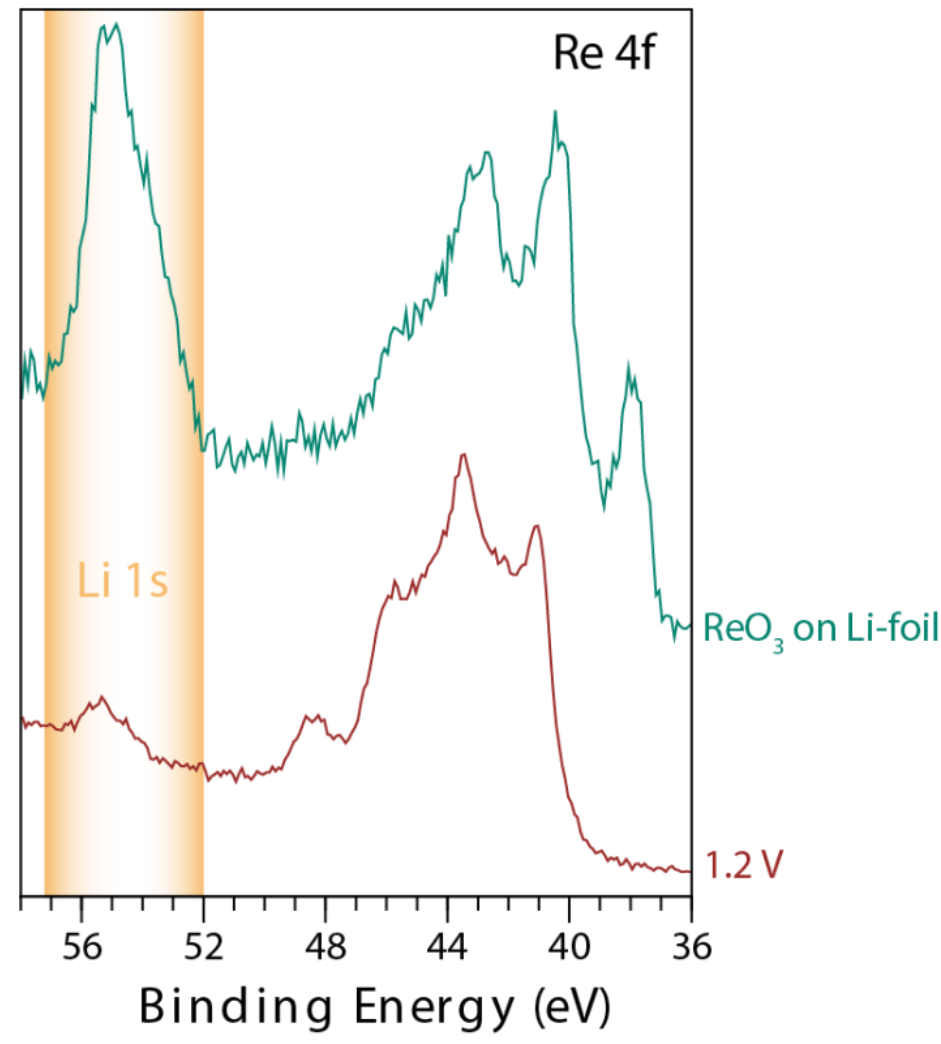

Figure S15. Re 4f narrow region XPS scans for $\mathrm{ReO}_{3} \mathrm{NCs}$ charged to $1.2 \mathrm{~V}$ (most reduced state, red curve) and those deposited on a cleaned Li-foil (green curve). The blue-green appearance of the NCs immediately converts to black signifying quick reduction by the Li metal. The Li 1 s regions are marked. 


\section{References}

1. Beattie, I. R.; Gilson, T. R.; Jones, P. J., Vapor Phase Vibrational Spectra for $\mathrm{Re}_{2} \mathrm{O}_{7}$ and the Infrared Spectrum of Gaseous $\mathrm{HReO}_{4}$. Molecular Shapes of $\mathrm{Mn}_{2} \mathrm{O}_{7}, \mathrm{Tc}_{2} \mathrm{O}_{7}$, and $\mathrm{Re}_{2} \mathrm{O}_{7}$. Inorg. Chem. 1996, 35 (5), 1301-1304.

2. Feinleib, J.; Scouler, W. J.; Ferretti, A., Optical Properties of the $\mathrm{Metal}^{\mathrm{ReO}} \mathrm{O}_{3}$ from 0.1 to 22 eV. Phys. Rev. 1968, 165 (3), 765-774.

3. Bohren, C. F.; Huffman, D. R., Absorption and Scattering of Light by Small Particles. Wiley: 2008.

4. Maier, S. A., Localized Surface Plasmons. In Plasmonics: Fundamentals and Applications, Maier, S. A., Ed. Springer US: New York, NY, 2007; pp 65-88.

5. Kreibig, U.; Vollmer, M., Optical properties of metal clusters. Springer: 1995.

6. Kedenburg, S.; Vieweg, M.; Gissibl, T.; Giessen, H., Linear refractive index and absorption measurements of nonlinear optical liquids in the visible and near-infrared spectral region. Opt. Mater. Express 2012, 2 (11), 1588-1611.

7. Ashcroft, N. W.; Mermin, N. D., Solid State Physics. Holt, Rinehart and Winston: 1976.

8. Momma, K.; Izumi, F., VESTA 3 for three-dimensional visualization of crystal, volumetric and morphology data. J. Appl. Crystallogr. 2011, 44 (6), 1272-1276. 
